\title{
RIEMANN CURVATURE OF A BOOSTED SPACETIME GEOMETRY
}

\author{
EMMANUELE BATTISTA \\ Dipartimento di Fisica, Complesso Universitario di Monte S. Angelo, Via Cintia Edificio 6, \\ 80126 Napoli, Italy \\ Istituto Nazionale di Fisica Nucleare, Sezione di Napoli, Complesso Universitario di Monte S. \\ Angelo, Via Cintia Edificio 6, 80126 Napoli, Italy \\ ebattista@na.infn.it \\ GIAMPIERO ESPOSITO \\ Istituto Nazionale di Fisica Nucleare, Sezione di Napoli, Complesso Universitario di Monte S. \\ Angelo, Via Cintia Edificio 6, 80126 Napoli, Italy \\ gesposit@na.infn.it \\ PAOLO SCUDELLARO \\ Dipartimento di Fisica, Complesso Universitario di Monte S. Angelo, Via Cintia Edificio 6, \\ 80126 Napoli, Italy \\ Istituto Nazionale di Fisica Nucleare, Sezione di Napoli, Complesso Universitario di Monte S. \\ Angelo, Via Cintia Edificio 6, 80126 Napoli, Italy \\ scud@na.infn.it \\ FRANCESCO TRAMONTANO \\ Dipartimento di Fisica, Complesso Universitario di Monte S. Angelo, Via Cintia Edificio 6, \\ 80126 Napoli, Italy \\ Istituto Nazionale di Fisica Nucleare, Sezione di Napoli, Complesso Universitario di Monte S. \\ Angelo, Via Cintia Edificio 6, 80126 Napoli, Italy \\ tramonta@na.infn.it
}

The ultrarelativistic boosting procedure had been applied in the literature to map the metric of Schwarzschild-de Sitter spacetime into a metric describing de Sitter spacetime plus a shock-wave singularity located on a null hypersurface. This paper evaluates the Riemann curvature tensor of the boosted Schwarzschild-de Sitter metric by means of numerical calculations, which make it possible to reach the ultrarelativistic regime gradually by letting the boost velocity approach the speed of light. Thus, for the first time in the literature, the singular limit of curvature, through Dirac's $\delta$ distribution and its derivatives, is numerically evaluated for this class of spacetimes. Moreover, the analysis of the Kretschmann invariant and the geodesic equation shows that the spacetime possesses a "scalar curvature singularity" within a 3 -sphere and it is possible to define what we here call "boosted horizon", a sort of elastic wall where all particles are surprisingly pushed away, as numerical analysis demonstrates. This seems to suggest that such "boosted geometries" are ruled by a sort of "antigravity effect" since all geodesics seem to refuse to enter the "boosted horizon" and are "reflected" by it, even though their initial conditions are aimed at driving the particles towards the "boosted horizon" itself. Eventually, the equivalence with the coordinate shift method is invoked in order to 
demonstrate that all $\delta^{2}$ terms appearing in the Riemann curvature tensor give vanishing contribution in distributional sense.

Keywords: boost, black hole, singularity

\section{Introduction}

The subject of gravitational fields generated by sources which move at the speed of light has been extensively studied in the literature because of its close connection to the topic of gravitational wavesa, whose direct detection remains extremely difficult, since one normally deals with a very weak signal. The first who dealt with this aspect of general relativity was Tolman in 1934 [1], who studied the gravitational field of light beams and pulses in the linearized theory. But it was only in 1971 that Aichelburg and Sexl 2] developed a method to describe the gravitational field associated to a massless point particle moving at the speed of light in Minkowski spacetime (i.e. the gravitational field from a single photon). In fact in Ref. 22 the authors first derive this field by solving the linearized Einstein field equations for a particle with rest mass $m$ moving uniformly with velocity $v$. Then they take the limit $v \rightarrow 1$ while the mass of the particle tends to zero in such a way that its energy remains finite. After that, they start with the full Einstein theory and the Schwarzschild metrib (the exact metric describing a particle at rest), which written in isotropic coordinates reads as

$$
\mathrm{d} s^{2}=\frac{(1-A)^{2}}{(1+A)^{2}} \mathrm{~d} t^{2}-(1+A)^{4}\left(\mathrm{~d} x^{2}+\mathrm{d} y^{2}+\mathrm{d} z^{2}\right)
$$

with $A=m / 2 r$ and $r^{2}=x^{2}+y^{2}+z^{2}$. Afterwards they apply to this metric a Lorentz transformation

$$
\begin{gathered}
\bar{t}=\left(1-v^{2}\right)^{-1 / 2}(t+v x), \\
\bar{x}=\left(1-v^{2}\right)^{-1 / 2}(x+v t), \\
\bar{y}=y, \\
\bar{z}=z,
\end{gathered}
$$

to obtain the gravitational field as seen by an observer moving uniformly with velocity $v$ relative to the mass. Once the limits $v \rightarrow 1$ and $m \rightarrow 0$ are taken, Aichelburg and Sexl obtain the remarkable result that both the linearized solution and the exact solution agree completely.

The method first developed by Aichelburg and Sexl is called in the literature "the boost of a metric". With this procedure it is possible to show that the gravitational field of a null source is nonvanishing on a plane containing the particle and

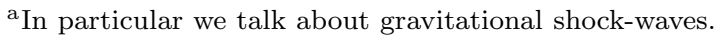

${ }^{\mathrm{b}}$ For a modern and innovative review of Schwarzschild solution see Ref. [3].
} 
orthogonal to the direction of motion, i.e. (asymmetric) plane-fronted gravitational waves. The Riemann curvature tensor is zero everywhere except on this plane, where it assumes a distributional nature. The intriguing fact is that the boosted metric in the ultrarelativistic regime $(v \rightarrow 1)$ has a new type of singularity, i.e. a distributional (Dirac-delta-like) singularity. The boosted ultrarelativistic metric obtained in Ref. 2] reads indeed as

$\mathrm{d} s^{2}=\mathrm{d} \bar{t}^{2}-\mathrm{d} \bar{x}^{2}-\mathrm{d} \bar{y}^{2}-\mathrm{d} \bar{z}^{2}-4 p\left\{(|\bar{t}-\bar{x}|)^{-1}-2 \delta\left(\bar{t}^{2}-\bar{x}^{2}\right) \log \sqrt{\bar{y}+\bar{z}}\right\}(\mathrm{d} \bar{t}-\mathrm{d} \bar{x})^{2}$,

with $p \equiv m / \sqrt{1-v^{2}}$. Thus, the gravitational field turns out to travel with the particle, being zero everywhere except at the hypersurface $\bar{t}=\bar{x}$. Moreover, as anticipated before, the Riemann tensor of (1.6) is zero everywhere except on the hypersurface $\bar{t}=\bar{x}$ and has nonvanishing components given by [2]

$$
\begin{gathered}
R_{0202}=4 p \delta(\bar{t}-\bar{x})\left[\frac{\bar{y}^{2}-\bar{z}^{2}}{\left(\bar{y}^{2}+\bar{z}^{2}\right)^{2}}+\pi \delta(\bar{y}) \delta(\bar{z})\right], \\
R_{0303}=4 p \delta(\bar{t}-\bar{x})\left[\frac{\bar{y}^{2}-\bar{z}^{2}}{\left(\bar{y}^{2}+\bar{z}^{2}\right)^{2}}-\pi \delta(\bar{y}) \delta(\bar{z})\right], \\
R_{0203}=-4 p \delta(\bar{t}-\bar{x}) \frac{2 \bar{y} \bar{z}}{\left(\bar{y}^{2}+\bar{z}^{2}\right)^{2}},
\end{gathered}
$$

with the other components related to the ones given above by symmetry. An important remark should be made at this point, since the Riemann tensor is perfectly defined as it contains the tensor product of Dirac's $\delta$ distributions (and not their multiplications). The only elements which are "poorly defined" in (1.7)-(1.9) are represented by the functions

$$
\frac{\bar{y}^{2}-\bar{z}^{2}}{\left(\bar{y}^{2}+\bar{z}^{2}\right)^{2}}
$$

and

$$
\frac{2 \bar{y} \bar{z}}{\left(\bar{y}^{2}+\bar{z}^{2}\right)^{2}}
$$

which are not locally integrable on the $(y, z)$-plane and, therefore, do not define, a priori, any distribution. Of course, their "regularization" (a la Gel'fand, see for example Ref. 4) is straightforward: the integration is understood in such a way that we first integrate over the set $y^{2}+z^{2}>\epsilon$ and then pass to the limit $\epsilon \rightarrow 0$.

In order to give a precise meaning to expressions (1.7)-(1.9), instead of transforming the Schwarzschild metric (1.1), the authors of Ref. 2 have applied the Lorentz transformations (1.2)-(1.5) directly to the components of the Riemann tensor and then they have investigated the regime $v \rightarrow 1$. In this way, with the help of tetrad formalism, Aichelburg and Sexl have obtained relations which are valid only for those spacetime points where $\bar{y}^{2}+\bar{z}^{2} \neq 0$. In particular, they obtain again the relations (1.7)-(1.9), but without the functions $\delta(\bar{y}) \delta(\bar{z})$ which vanish because 
of the condition $\bar{y}^{2}+\bar{z}^{2} \neq 0$. This fact shows that on the hypersurface $\bar{x}=\bar{t}$ the Riemann tensor has a $\delta$-like singularity and is exactly of Petrov type $N$ (i.e. all four principal null directions of the Weyl spinor, describing the Weyl conformal curvature, coincide).

Years after the work by Aichelburg and Sexl, more general impulsive waves were obtained by boosting other black hole spacetimes with rotation, charge and a cosmological constant $8 / 5 / 6 / 7 / 8 / 9 / 10 / 11 / 1213$. The technique of boosting a spacetime metric in fact has a lot of applications in theoretical physics. The work in Ref. 14, for instance, shows that the black hole formation caused by the collision of two particles with large relative velocity $(v \rightarrow 1)$, and considered in the rest frame of one of the particles, involves the concept of boosted metric: the gravitational field of the other particle is described by the ultrarelativistic boosted Schwarschild-de Sitter metric. Moreover, the collisions of shock-waves and heavy ions as well as the entropy that is consequently produced [15] appeal to the boost procedure, also in the context of higher dimensions [16] and branes 17/1819/20. Furthermore, it is possible to study the formation of marginally trapped surfaces in the head-on collision both of two shock-waves 21] and of two ultrarelativistic charged particles [22] in de Sitter space by using the procedure of boosting a metric, since for example in the latter case the metric of the two charges is obtained by boosting ReissnerNordström-de Sitter spacetime to the speed of light, while with similar arguments it is shown in Ref. [23] that the collision of two Reissner-Nordström gravitational shock-waves in anti-de Sitter space prevents the formation of marginally trapped surfaces of Penrose type. Finally, the concept of a boosted metric can be used as a tool to describe (de Sitter) spacetime from a quantum point of view 24].

Our main attention here will be devoted to the work in Refs. 7], [13] where it has been shown in detail how to map, through a boosting procedure, the Schwarzschildde Sitter metric

$$
\mathrm{d} s^{2}=-\left(1-\frac{2 m}{r}-\frac{r^{2}}{a^{2}}\right) \mathrm{d} t^{2}+\frac{\mathrm{d} r^{2}}{\left(1-\frac{2 m}{r}-\frac{r^{2}}{a^{2}}\right)}+r^{2}\left(\mathrm{~d} \theta^{2}+\sin ^{2} \theta \mathrm{d} \phi^{2}\right),
$$

into the highly singular form (with $v \rightarrow 1$ )

$$
\begin{aligned}
\mathrm{d} s^{2}= & -\mathrm{d} Y_{0}^{2}+\mathrm{d} Y_{1}^{2}+\mathrm{d} Y_{2}^{2}+\mathrm{d} Y_{3}^{2}+\mathrm{d} Y_{4}^{2} \\
& +4 p\left[-2+\frac{Y_{4}}{a} \log \left(\frac{a+Y_{4}}{a-Y_{4}}\right)\right] \delta\left(Y_{0}+Y_{1}\right)\left(\mathrm{d} Y_{0}+\mathrm{d} Y_{1}\right)^{2},
\end{aligned}
$$

where the first line describes de Sitter space viewed as a four-dimensional hyperboloid of radius $a$ having equation

$$
\left(Y_{0}\right)^{2}=-a^{2}+\left(Y_{1}\right)^{2}+\left(Y_{2}\right)^{2}+\left(Y_{3}\right)^{2}+\left(Y_{4}\right)^{2},
$$

embedded into flat five-dimensional space, while the second line of (1.13) describes a shock-wave singularity located on the null hypersurface having equations

$$
Y_{0}+Y_{1}=0
$$

${ }^{\mathrm{c}}$ For the manifestly four-dimensional form of metric 1.13 see Appendix Appendix B 


$$
\left(Y_{2}\right)^{2}+\left(Y_{3}\right)^{2}+\left(Y_{4}\right)^{2}-a^{2}=0,
$$

equation (1.16) being obtained by the joint effect of the hyperboloid constraint (1.14) and the Dirac-delta condition (1.15). Since the metric is turned into a mathematical object having distributional nature, the usual spacetime picture is no longer valid, but it would be very interesting to evaluate the effect of these shock-wave singularities on curvature. The great revolution introduced by Einstein's theory consists in fact in viewing the gravitational field as the curvature of spacetime. Such a curvature is directly coupled to the energy and momentum of whatever matter and radiation are present, as specified by the Einstein field equations whose content states that "the matter and the energy say to the spacetime how to curve, and the curvature of spacetime says to the matter how to move" 25. Thus, one of the most important objects of the theory of the gravitational field is the Riemann tensor, since it is an intrinsic object that catches in an elegant and covariant way the features of spacetime curvature. Therefore it could be of great physical importance to evaluate the Riemann tensor for this type of geometries, i.e. "the boosted geometries". (To fully appreciate the importance of this tensor see Appendix Appendix A.

Since "gravitation is a manifestation of spacetime curvature, and curvature shows up in the deviation of one geodesic from a nearby geodesic" [25, the concept of spacetime curvature is directly related to the geodesic completeness of spacetime, as we say that a spacetime manifold is geodesically complete if any geodesic can be extended to arbitrary values of the affine parameter. Thus, knowledge of the Riemann curvature tensor is an essential step towards the description of topological features of spacetime and this motivates the effort we made in calculating the Riemann tensor for the boosted Schwarschild-de Sitter metric.

We stress that the definitions (A.1)- A.5 are given in terms of objects that, unlike the ones we will handle, have no distributional singularities (cfr (1.13)). Thus, in this article we are interested in a sort of generalization of the usual concept of Riemann tensor, which enlarges the notion of curvature, i.e. what we call the "boosted Riemann tensor", with a particular interest in the ultrarelativistic regime, where distributional singularities show up. By virtue of the high difficulty of dealing with the metric (1.13), we decided to start from its low-velocity limit and then to reach the ultrarelativistic regime via numerical calculations. For this purpose, Sec. II evaluates the procedure to obtain the boosted Schwarzschild-de Sitter metric in manifestly four-dimensional form. Then, it is shown that the basis defined by the boost procedure is a coordinate basis, a property that greatly simplifies the calculations performed. In Sec. III the analysis of both the Kretschmann invariant and the geodesic equation allows us to characterize the features of curvature.

An important question arises while dealing with Secs. II-III, i.e. how to deal with the Riemann curvature tensor when it has terms proportional to $\delta^{2}$. In fact from (1.13) it is easy to understand that the Riemann tensor has got terms involving the products of two Dirac's $\delta$ distributions (a formal method to cope with multi- 
E. Battista, G. Esposito, P. Scudellaro, F. Tramontano

plication of distributions can be found in Ref. [26]). This means that the "boosted Riemann tensor" of the "boosted geometry" we are going to describe is in principle not defined. Anyway, we will be able to show that the $\delta^{2}$ terms appearing in the "boosted Riemann tensor" vanish in a distributional sense. Unlike the (rather simple) example discussed in Ref. 2, we will achieve this point in a more difficult way, since the high difficulty of metric (1.13) makes it quite impossible to write down explicitly all the boosted Riemann tensor components, as anticipated above. For this reason in Sec. IV we will make use of an equivalent method to describe the gravitational shock-wave of a massless particle, i.e. the coordinate shift method 2728, (or the scissors-and-paste method by Penrose [29]). The equivalence of this method and the boosting procedure has been demonstrated by the authors of Ref. [27, where it is explicitly shown that with the new approach it is possible to recover the results of Aichelburg and Sexl. By exploiting this equivalence between the two methods, we will be able to show in which sense the $\delta^{2}$ terms appearing in the Riemann tensor of metric (1.13) can be seen as vanishing, leading to a well defined spacetime function (in the sense of distributions). Concluding remarks and open problems are presented in Sec. V.

\section{The "boosted" Riemann curvature tensor}

Following Refs. [7, [13] we can express a de Sitter spacetime in four dimensions as a four-dimensional hyperboloid of radius $a$ embedded in five-dimensional Minkowski spacetime having metric

$$
\mathrm{d} s_{M}^{2}=-\mathrm{d} Z_{0}^{2}+\mathrm{d} Z_{1}^{2}+\mathrm{d} Z_{2}^{2}+\mathrm{d} Z_{3}^{2}+\mathrm{d} Z_{4}^{2},
$$

with coordinates satisfying the hyperboloid constraint

$$
a^{2}=-\left(Z_{0}\right)^{2}+\left(Z_{1}\right)^{2}+\left(Z_{2}\right)^{2}+\left(Z_{3}\right)^{2}+\left(Z_{4}\right)^{2} .
$$

By exploiting the relations between the $Z_{i}(i=0,1,2,3,4)$ coordinates and the spherical static coordinates $(t, r, \theta, \phi)$

$$
\begin{gathered}
Z_{0} \equiv \sqrt{a^{2}-r^{2}} \sinh (t / a), \\
Z_{1} \equiv r \cos \theta, \\
Z_{2} \equiv r \sin \theta \cos \phi, \\
Z_{3} \equiv r \sin \theta \sin \phi, \\
Z_{4} \equiv \pm \sqrt{a^{2}-r^{2}} \cosh (t / a),
\end{gathered}
$$

and on defining

$$
f^{2} \equiv a^{2}-r^{2}=\left(Z_{4}\right)^{2}-\left(Z_{0}\right)^{2}
$$




$$
\begin{gathered}
F_{m} \equiv 1-\frac{2 a^{2} m}{f^{2} r}-\frac{a^{2} / r^{2}}{\left(1-\frac{2 a^{2} m}{f^{2} r}\right)}, \\
Q \equiv 1+\frac{2\left(Z_{0}\right)^{2}}{f^{2}}
\end{gathered}
$$

we can express the Schwarschild-de Sitter metric (1.12) in the form

$$
\mathrm{d} s^{2}=h_{00} \mathrm{~d} Z_{0}^{2}+h_{44} \mathrm{~d} Z_{4}^{2}+2 h_{04} \mathrm{~d} Z_{0} \mathrm{~d} Z_{4}+\mathrm{d} Z_{1}^{2}+\mathrm{d} Z_{2}^{2}+\mathrm{d} Z_{3}^{2},
$$

where

$$
\begin{gathered}
h_{00} \equiv-\frac{1}{2}(Q-1) F_{m}-\left(1-\frac{2 a^{2} m}{f^{2} r}\right)-\frac{\left(Z_{0}\right)^{2}}{r^{2}} \\
h_{44} \equiv-\frac{1}{2}(Q+1) F_{m}+\left(1-\frac{2 a^{2} m}{f^{2} r}\right)-\frac{\left(Z_{4}\right)^{2}}{r^{2}} \\
h_{04} \equiv \frac{Z_{0} Z_{4}}{f^{2}} F_{m}+\frac{Z_{0} Z_{4}}{r^{2}} .
\end{gathered}
$$

At this stage, we introduce a Lorentz boost in the $Z_{1}$-direction by defining a new set of coordinates independent of $v$, i.e. the $Y_{i}$ coordinates, such that (hereafter $\left.\gamma \equiv 1 / \sqrt{1-v^{2}}\right)$

$$
\begin{gathered}
Z_{0}=\gamma\left(Y_{0}+v Y_{1}\right), \\
Z_{1}=\gamma\left(v Y_{0}+Y_{1}\right), \\
Z_{2}=Y_{2}, \quad Z_{3}=Y_{3}, \quad Z_{4}=Y_{4} .
\end{gathered}
$$

Thus, starting from (2.11) jointly with (2.15)-(2.17) we eventually obtain the boosted Schwarzschild-de Sitter metric

$$
\begin{aligned}
\mathrm{d} s^{2}= & \gamma^{2}\left(h_{00}+v^{2}\right) \mathrm{d} Y_{0}^{2}+\gamma^{2}\left(1+v^{2} h_{00}\right) \mathrm{d} Y_{1}^{2}+\mathrm{d} Y_{2}^{2}+\mathrm{d} Y_{3}^{2}+h_{44} \mathrm{~d} Y_{4}^{2} \\
& +2 v \gamma^{2}\left(1+h_{00}\right) \mathrm{d} Y_{0} \mathrm{~d} Y_{1}+2 \gamma h_{04} \mathrm{~d} Y_{0} \mathrm{~d} Y_{4}+2 v \gamma h_{04} \mathrm{~d} Y_{1} \mathrm{~d} Y_{4}
\end{aligned}
$$

whose singular ultrarelativistic limit is expressed by (1.13). Thus, we can interpret (2.18) as the low-velocity limit of (1.13).

The spacetime metric (2.18) is apparently expressed by a $5 \times 5$ matrix while the original metric (1.12) is expressed through 4 local coordinates $t, r, \theta, \phi$. Hence also the metric (2.18) should be eventually expressed through 4 coordinates only, if one wants to arrive at a formula for the curvature, since our reference spacetime remains four-dimensional. To restore the usual four-dimensional form of the metric, we have to exploit the constraint (2.2) expressed in terms of $Y_{i}$ coordinates, i.e. Eq. (1.14). By virtue of this condition we can write

$$
Y_{0}=\sqrt{-a^{2}+\left(Y_{1}\right)^{2}+\left(Y_{2}\right)^{2}+\left(Y_{3}\right)^{2}+\left(Y_{4}\right)^{2}} \equiv \sqrt{\sigma\left(Y_{\mu}\right)},
$$


E. Battista, G. Esposito, P. Scudellaro, F. Tramontano

$$
d Y_{0}=\frac{\sum_{\mu=1}^{4} Y_{\mu} d Y_{\mu}}{\sqrt{\sigma\left(Y_{\mu}\right)}},
$$

and eventually, using (2.19) and (2.20), we obtain the manifestly four-dimensional form of the boosted metric (2.18), which can be expressed by the relations

$$
\begin{aligned}
& g_{11}=\frac{\gamma^{2}\left(h_{00}+v^{2}\right)}{\sigma}\left(Y_{1}\right)^{2}+\gamma^{2}\left(1+v^{2} h_{00}\right)+\frac{2 v \gamma^{2}\left(1+h_{00}\right)}{\sqrt{\sigma}} Y_{1}, \\
& g_{22}=\frac{\gamma^{2}\left(h_{00}+v^{2}\right)}{\sigma}\left(Y_{2}\right)^{2}+1, \\
& g_{33}=\frac{\gamma^{2}\left(h_{00}+v^{2}\right)}{\sigma}\left(Y_{3}\right)^{2}+1, \\
& g_{44}=\frac{\gamma^{2}\left(h_{00}+v^{2}\right)}{\sigma}\left(Y_{4}\right)^{2}+h_{44}+\frac{2 \gamma h_{04}}{\sqrt{\sigma}} Y_{4}, \\
& g_{12}=\frac{\gamma^{2}\left(h_{00}+v^{2}\right)}{\sigma} Y_{1} Y_{2}+\frac{v \gamma^{2}\left(1+h_{00}\right)}{\sqrt{\sigma}} Y_{2}, \\
& g_{13}=\frac{\gamma^{2}\left(h_{00}+v^{2}\right)}{\sigma} Y_{1} Y_{3}+\frac{v \gamma^{2}\left(1+h_{00}\right)}{\sqrt{\sigma}} Y_{3}, \\
& g_{14}=\frac{\gamma^{2}\left(h_{00}+v^{2}\right)}{\sigma} Y_{1} Y_{4}+\frac{v \gamma^{2}\left(1+h_{00}\right)}{\sqrt{\sigma}} Y_{4}+\frac{\gamma h_{04}}{\sqrt{\sigma}}+v \gamma h_{04}, \\
& g_{23}=\frac{\gamma^{2}\left(h_{00}+v^{2}\right)}{\sigma} Y_{2} Y_{3} \\
& g_{24}=\frac{\gamma^{2}\left(h_{00}+v^{2}\right)}{\sigma} Y_{2} Y_{4}+\frac{\gamma h_{04}}{\sqrt{\sigma}} Y_{2}, \\
& g_{34}=\frac{\gamma^{2}\left(h_{00}+v^{2}\right)}{\sigma} Y_{3} Y_{4}+\frac{\gamma h_{04}}{\sqrt{\sigma}} Y_{3} .
\end{aligned}
$$

Having obtained the formulas (2.21)-(2.30), we can evaluate the RiemannChristoffel symbols and consequently the Riemann curvature tensor of the boosted Schwarzschild-de Sitter metric by using the familiar relations of classical general relativity. The most general form of Riemann-Christoffel symbols reads as follows [25] ( $a, b, c$ being abstract indices):

$$
\Gamma_{a b c}=\frac{1}{2}\left(g_{a b, c}+g_{a c, b}-g_{b c, a}+c_{a b c}+c_{a c b}-c_{b c a}\right),
$$

where the "commutation coefficients" $c_{a b c}$ are defined by

$$
\left[\mathbf{e}_{b}, \mathbf{e}_{c}\right] \equiv c_{b c}{ }^{a} \mathbf{e}_{a}
$$


with $\left\{\mathbf{e}_{a}\right\}$ being any noncoordinate basis. Last, the components of the Riemann tensor are given by

$$
R_{b c d}^{a}=\Gamma_{b d, c}^{a}-\Gamma_{b c, d}^{a}+\Gamma_{b d}^{e} \Gamma_{e c}^{a}-\Gamma_{b c}^{e} \Gamma_{e d}^{a}-\Gamma_{b e}^{a} c_{c d}^{e} .
$$

We can somewhat simplify the relations (2.31), (2.33) in the case in which $\left\{\frac{\partial}{\partial Y_{\mu}}\right\}$ ( $\mu$ being a coordinate index such that $\mu=1,2,3,4$ ) is a coordinate basis. As we know, the static spherical basis $(t, r, \theta, \phi)$ is indeed a coordinate basis. Bearing in mind definitions (2.3) (2.7), the Jacobian of the transformation between the spherical coordinates and the $\left\{\frac{\partial}{\partial Z_{\mu}}\right\}$ is expressed by

$$
J_{\mu}{ }^{\lambda}=\left(\begin{array}{cccc}
0 & \cos \theta & -r \sin \theta & 0 \\
0 & \sin \theta \cos \phi & r \cos \theta \cos \phi-r \sin \theta \sin \phi \\
0 & \sin \theta \sin \phi & r \cos \theta \sin \phi & r \sin \theta \cos \phi \\
\frac{\sqrt{a^{2}-r^{2}}}{a} \sinh (t / a) & \frac{-r}{\sqrt{a^{2}-r^{2}}} \cosh (t / a) & 0 & 0
\end{array}\right) \text {, }
$$

while the inverse Jacobian reads as

$$
\begin{aligned}
& \left(J^{-1}\right)_{\lambda}{ }^{\mu}= \\
& \left(\begin{array}{cccc}
\frac{a r \cos \theta \operatorname{coth}(t / a)}{\left(a^{2}-r^{2}\right)} & \frac{a r \cos \phi \sin \theta \operatorname{coth}(t / a)}{\left(a^{2}-r^{2}\right)} & \frac{a r \sin \theta \sin \phi \operatorname{coth}(t / a)}{\left(a^{2}-r^{2}\right)} & \frac{a(\sinh (t / a))^{-1}}{\sqrt{a^{2}-r^{2}}} \\
\cos \theta & \cos \phi \sin \theta & \sin \theta \sin \phi & 0 \\
-\sin \theta / r & \cos \theta \cos \phi / r & \cos \theta \sin \phi / r & 0 \\
0 & -\frac{\sin \phi}{r \sin \theta} & \frac{\cos \phi}{r \sin \theta} & 0
\end{array}\right) .
\end{aligned}
$$

By virtue of (2.34) and (2.35), if we adopt the concise notation $x_{\lambda} \equiv(t, r, \theta, \phi)$ we can write

$$
\frac{\partial}{\partial Z_{\mu}}=\left(J^{-1}\right)_{\lambda}^{\mu} \frac{\partial}{\partial x_{\lambda}}
$$

and, by exploiting the fact that $\left\{\frac{\partial}{\partial x_{\lambda}}\right\}$ is a coordinate basis, after a lengthy calculation we arrive at the conclusion that also the basis $\left\{\frac{\partial}{\partial Z_{\mu}}\right\}$ is a coordinate basis, or in other words we have that

$$
\left[\frac{\partial}{\partial Z_{\mu}}, \frac{\partial}{\partial Z_{\lambda}}\right]=0
$$

The relations (2.15)-(2.17) for the boost show that the transformations between $Z_{\mu}$ and $Y_{\mu}$ are linear, therefore we can easily conclude that

$$
\left[\frac{\partial}{\partial Y_{\mu}}, \frac{\partial}{\partial Y_{\lambda}}\right]=0
$$


hence the basis $\left\{\frac{\partial}{\partial Y_{\mu}}\right\}$ is a coordinate basis as well.

This means that we can evaluate the Riemann-Christoffel symbols and the Riemann curvature tensor for the boosted spacetime metric (2.21)-(2.30) by setting $c_{a b c}=0$ in the relations (2.31), (2.33). Nevertheless, these relations are still too complicated to be computed analytically, and therefore a numerical calculation has been necessary. Formulas (2.21)-(2.30) show indeed that we are dealing with a spacetime metric represented by a $4 \times 4$ matrix whose elements are given by some complicated nonvanishing functions of the $Y_{\mu}$ coordinates. That is why we first tried to compute the Riemann curvature tensor analytically in terms of tetrads (see Appendix Appendix B before realizing that even this solution was far too complicated. Thus, the only way we had to compute the Riemann-Christoffel symbols and the Riemann tensor was represented by numerical calculations. In this way we can evaluate the behavior of spacetime curvature also in the ultra-relativistic regime, which is the one we are mainly interested in, by letting the velocity defined by the boost relations (2.15)-(2.17) approach gradually the speed of light.

In what follows we discuss the results of our computation mainly by studying curvature invariants and the behavior of geodesics in our reference spacetime. We in fact think that these features represent the best tools to describe physically the concept of spacetime curvature.

\section{The Kretschmann invariant}

Intuitively, a spacetime singularity is a "place" where the curvature "blows up" 30] or, by analogy with electrodynamics, a point where the metric tensor is either not defined or not suitably differentiable [31. Regrettably, both these statements are not rigorous definitions that can characterize the concept of singularity. First of all, since in general relativity we do not know the manifold and the metric structure in advance (they are solutions of Einstein field equations), we are not able to give a physical sense to the notion of an event until we solve Einstein equations, and hence the idea of a singularity as a "place" has not a satisfactory meaning. Moreover, also the notion of curvature becoming larger and larger as a general criterion for singularities has pathological problems. In fact, the bad behavior of components or derivatives of the Riemann tensor could be ascribed to the coordinate or tetrad basis used. To avoid this problem, one might examine scalar curvature invariants constructed from the Riemann tensor or its covariant derivatives, which in some cases can completely characterize the spacetime (see Refs. 3233 for further details). However, even if the value of some scalar invariants is unbounded, curvature might blow up only "as one goes to infinity", a case that we would interpret as a singularity-free spacetime [30]. Furthermore, spacetimes may be singular without any bad behavior of the curvature tensor (the so-called "conical singularities" 30]). Lastly, the bad behavior of the metric tensor at some spacetime points cannot be a way to define singularities, as one could always cut out such 
points and hence the remaining manifold, representing the whole spacetime, would turn out to be nonsingular.

A more satisfactory idea to define singularities is to use the notion of incompleteness of timelike geodesics, i.e. geodesics which are inextendible in at least one direction and hence have only a finite range of affine parameter. This has the immediate physical interpretation that there exist freely moving observers or particles whose histories did not exist after (or before) a finite interval of proper time. Although the physical meaning of affine parameter on null geodesics is different from the case of timelike geodesics, we could also regard null geodesic incompleteness as a good criterion to define spacetime singularities. Thus, timelike and null geodesic completeness are minimum conditions for spacetime to be considered singularityfree 31. However, as there are examples of geodesically complete spacetimes which contain an inextendible timelike curve of bounded acceleration and finite length 34, we should generalize the concept of affine parameter to all $C^{1}$ curves, no matter whether they are geodesics or not. This fact is linked to the concept of bcompleteness (short for bundle completeness), which we shortly describe following Refs. 3135, in Appendix Appendix C

Therefore, we can classify a singularity represented by the presence of at least one incomplete geodesic according to whether [30]

(1) a curvature invariant blows up along a geodesic ("scalar curvature singularity"),

(2) a component of the Riemann tensor or its covariant derivatives in a parallelly propagated tetrad blows up along a geodesic ("parallelly propagated curvature singularity"),

(3) no such invariant or component blows up ("noncurvature singularity").

We can therefore understand the importance of scalar curvature invariants in the analysis of spacetime singularities. Being coordinate independent, curvature invariants can describe the size of curvature and its growth along timelike curves, and can also characterize curvature singularities [36], while providing important information about the nature of singularities. For example, in the case of Schwarzschild metric, which can be obtained from (1.12) if we put $a=\infty$ (for an unambiguous definition of the notion of limit applied to spacetimes see Ref. [37]), the Kretschmann invariant (i.e. the Riemann tensor squared) is such that

$$
R^{\alpha \beta \gamma \delta} R_{\alpha \beta \gamma \delta}=48 m^{2} / r^{6},
$$

in agreement with the fact that in all coordinate systems the real singularity is located only at $r=0$ and not also at $r=2 M$ (i.e. the event horizon).

In order to study the features of the Riemann curvature of spacetime described by the metric (1.13), we therefore decided to plot the Kretschmann invariant at different values of boost velocity $v$ and study the geodesic equation

$$
\ddot{Y}^{\mu}(s)+\Gamma_{\nu \lambda}^{\mu} \dot{Y}^{\nu}(s) \dot{Y}^{\lambda}(s)=0,
$$


$s$ being the affine parameter of the geodesic having parametric equation $Y^{\mu}=$ $Y^{\mu}(s)$.

From the analysis of the Kretschmann invariant we found that it is not defined unless the inequality (hereafter, numerical values of $Y$ coordinates have downstairs indices, to be consistent with the notation in Sec. II)

$$
\left(Y_{1}\right)^{2}+\left(Y_{2}\right)^{2}+\left(Y_{3}\right)^{2}+\left(Y_{4}\right)^{2}>a^{2},
$$

is satisfied. Hence, we see that the hyperboloid constraint, condition (1.14), allows us to define a 3 -sphere of radius $a$ where the Kretschmann invariant is not defined. This peculiar feature of our "boosted spacetime geometry" is indeed obvious if we look at formulas (2.21)-(2.30), as here the quantities $\sigma$ and $\sqrt{\sigma}$ always appear at the denominator of the expressions of the metric tensor $g_{\mu \nu}$, which means that the metric is defined only if the inequality (3.3) holds. Moreover, it is possible to derive Eq. (3.3) in the regime $v<1$ from the analysis of the Kretschmann invariant for the Schwarzschild-de Sitter metric (1.12). In fact for (1.12) the Kretschmann invariant reads as

$$
R^{\alpha \beta \gamma \delta} R_{\alpha \beta \gamma \delta}=24\left(\frac{1}{a^{4}}+\frac{2 m^{2}}{r^{6}}\right),
$$

which reduces to (3.1) in the limit $a=\infty$. Therefore, it follows immediately from (3.4) that the Schwarzschild-de Sitter metric (1.12) has an unique singularity located at $r=0$. Equation (E.9) clearly shows that the condition $r=0$ leads to

$$
\sqrt{\gamma^{2}\left(v \sqrt{\sigma}+Y_{1}\right)^{2}+\left(Y_{2}\right)^{2}+\left(Y_{3}\right)^{2}}=0,
$$

which, being defined by the sum of squared quantities, in turns implies that

$$
\left\{\begin{array}{l}
v \sqrt{\sigma}+Y_{1}=0 \\
Y_{2}=0 \\
Y_{3}=0
\end{array}\right.
$$

Thus, because of the presence of the term $\sqrt{\sigma}$, the condition $r=0$ is equivalent to (3.6), provided that $\sigma \geq 0$. If we now bear in mind that (2.21) -(2.30) prevent $\sigma$ from vanishing, we can conclude that the only possible choice is $\sigma>0$, which is equivalent to (3.3). In other words, the presence of the 3-sphere where the Kretschmann invariant is not defined follows directly from the condition $r=0$ which makes the curvature invariant (3.4) diverge. This fact can be interpreted as a hint indicating that this 3-sphere could represent a singularity of our "boosted geometry". Eventually, if we interpret $Y_{0}$ as the time coordinate (see (2.15)), we can view (3.3) as a condition on time.

In the $Y_{1}-Y_{2}$ plane this 3 -sphere becomes the circle with center at $Y_{1}=Y_{2}=$ 0 and radius $a$ of Fig. 1, which represents a contour plot of the Kretschmann invariant, i.e. a plot where each different color corresponds to different values of the Kretschmann invariant and these values increase as we approach this circle.

Another interesting feature of "boosted geometries" that we have found consists in the presence of a sort of barrier surrounding the 3 -sphere, which we may 


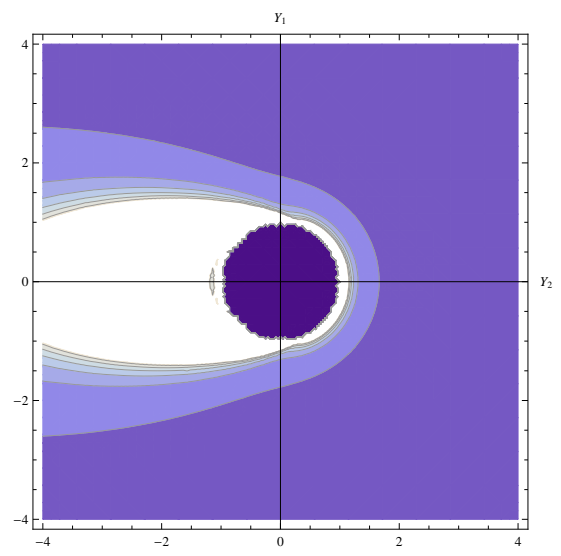

Fig. 1. Contour plot of the Kretschmann invariant numerically obtained with the following values of parameters: $a=1, m=0.1, Y_{3}=Y_{4}=0$ and $v=0.99$. The dark purple zone represents the circle of radius $a$ where the Kretschmann invariant is not defined.

call "boosted horizon", in the sense that all geodesics, despite maintaining their completeness condition, are surprisingly pushed away from it d We have also discovered that the extension of the "boosted horizon" depends on the boost velocity $v$, as we will shortly see. Since we have found that all geodesics are complete, according to standard definitions of general relativity the "boosted horizon" is not a singularity but, as we will show, it seems to be a sort of elastic wall which is hit by all particles before they get away. We have observed this effect numerically, by varying initial conditions of (3.2) and the boost velocity $v$, so as to reproduce different physical situations. Figures 2 and 3 indeed represent one among the many situations analyzed which witness this "antigravity" effect. Figures 2 and 3 show in fact a particle initially lying on the $Y_{1}=0$ line of Fig. 1 and having an initial velocity directed toward the region where the Kretschmann invariant is not defined. Strikingly, the solution "refuses" to be attracted by the 3 -sphere but, regardless of its initial velocity, the particle always arrives at a certain point and then it goes away from it, as if an elastic wall were present. We propose to call this elastic wall "boosted horizon". The position of such a "boosted horizon" is independent of the initial velocity of the particle, but depends only on the boost velocity $v$. In fact, bearing in mind Fig. 1, both for particles coming from "above" (i.e. particles initially lying on the positive half-line $Y_{2}>0, Y_{1}=0$ and with $\left.Y_{2}^{\prime}(0)<0\right)$ and for those coming from "below" (i.e. particles initially lying on the negative half-line $Y_{2}<0, Y_{1}=0$ and with $\left.Y_{2}^{\prime}(0)>0\right)$, the position of the "boosted horizon" does not

\footnotetext{
dMore precisely, one defines an "event horizon" as the boundary of the causal past of future null infinity 31. In the ultrarelativistic regime we cannot say if this concept is still valid and hence we talk about "boosted horizon" as the surface of spacetime surrounding the 3-sphere of radius $a$ where all geodesics, despite being complete, are pushed away.
} 
change, as Tab. 1 1shows. We have numerically checked, for each line of Tab. 1, that

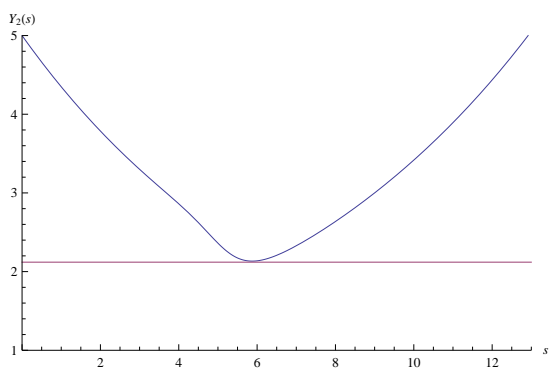

Fig. 2. Numerical solution of Eq. (3.2) for the function $Y_{2}(s)$ obtained in the $Y_{1}-Y_{2}$ plane and with initial conditions $Y_{1}(0)=Y_{3}(0)=Y_{4}(0)=0, Y_{2}(0)=5, Y_{1}^{\prime}(0)=Y_{3}^{\prime}(0)=Y_{4}^{\prime}(0)=0$ and $Y_{2}^{\prime}(0)=-0.7$. The values of parameters are $a=1, m=0.1$ and $v=0.9$. It is possible to see an "antigravity effect", since the function $Y_{2}(s)$ is pushed away from the "boosted horizon", which is represented by the horizontal line located at $Y_{2}=2.12$.

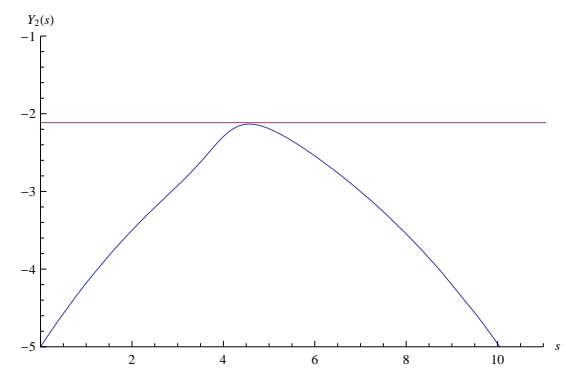

Fig. 3. Numerical solution of Eq. (3.2) for the function $Y_{2}(s)$ obtained in the $Y_{1}-Y_{2}$ plane and with initial conditions $Y_{1}(0)=Y_{3}(0)=Y_{4}(0)=0, Y_{2}(0)=-5, Y_{1}^{\prime}(0)=Y_{3}^{\prime}(0)=Y_{4}^{\prime}(0)=0$ and $Y_{2}^{\prime}(0)=0.9$. The values of parameters are $a=1, m=0.1$ and $v=0.9$. The function $Y_{2}(s)$ initially moves toward the "boosted horizon", i.e. the horizontal line at $Y_{2}=-2.12$, but then it is pushed away.

the minimum distance of the particle from the boundary of the 3-sphere is always bigger than its radius $a$, independently of the particle initial velocity. This means that the "boosted horizon" is always outside the 3 -sphere. For example, we find that, when the boost velocity $v=0.5$, the minimum distance $d_{m}=3.1$ when $a=1$, and it decreases monotonically as $v$ increases or decreases, reaching a minimum value of order $1.05 \div 1.10$.

The situation becomes somewhat intriguing when the particle lies initially on the $Y_{2}=0$ line (see Fig. 1). In fact, in the cases in which the particle lies initially on the positive half-line $Y_{1}>0, Y_{2}=0$, it always manages to hit the 3 -sphere where the Kretschmann invariant is not defined, even if its initial velocity is extremely 


\begin{tabular}{|l|c|}
\hline boost velocity & $\begin{array}{c}\text { "boosted horizon" location } \\
\left(Y_{2} \text { coordinate }\right)\end{array}$ \\
\hline 0.9995 & \pm 1.02 \\
0.9992 & \pm 1.02 \\
0.9991 & \pm 1.02 \\
0.999 & \pm 1.02 \\
0.99 & \pm 1.41 \\
0.9 & \pm 2.12 \\
0.8 & \pm 2.33 \\
0.7 & \pm 2.43 \\
0.6 & \pm 2.48 \\
0.5 & \pm 2.48 \\
0.4 & \pm 2.42 \\
0.3 & \pm 2.42 \\
0.2 & \pm 2.34 \\
0.1 & \pm 2.19 \\
0.01 & \pm 1.52 \\
0.00155 & \pm 1.00 \\
0.001 & \pm 0.88 \\
0.0001 & \pm 0.27 \\
\hline
\end{tabular}

Table 1. Location of the "boosted horizon" as a function of the boost velocity $v$. The positive sign refers to particles coming from "above" and the negative to those coming from "below". The values of parameters are $a=1$ and $m=0.1$.

low, as we can see from Fig. 4. After the particle reaches the 3-sphere, its geodesic is not defined anymore and hence, according to definitions given above and those of Appendix Appendix C, we can conclude that the 3-sphere of equation $\left(Y_{1}\right)^{2}+$ $\left(Y_{2}\right)^{2}+\left(Y_{3}\right)^{2}+\left(Y_{4}\right)^{2}=a^{2}$ defines a "scalar curvature singularity" for our "boosted geometry", as we have guessed before.

When the particle lies initially on the negative half-line $Y_{1}<0, Y_{2}=0$, its geodesic is not defined even before it reaches the 3 -sphere (see Fig. 怔). This means that another "scalar curvature singularity" exists. Its position depends only on the boost velocity $v$ and not on the particle initial velocity. In any case, numerical analysis shows that this kind of singularities exists only if the particle lies initially on the $Y_{2}=0$ line. We have repeated the same analysis also by putting $Y_{1}=Y_{2}=0$ in the relations defining the curvature, i.e. in the $Y_{3}-Y_{4}$ plane, and we have found the same "antigravity effect" of the previous cases, as shown in Figs. 6 and 7. which represent some examples among the many situations numerically analyzed. Interestingly, in this case we have found no "scalar curvature singularities".

In the ultrarelativistic regime $(v=0.9999)$ the "antigravity effects" are still present but, as is clear from Tab. 1 the position of the boosted horizon tends to that of the singularity 3 -sphere. 


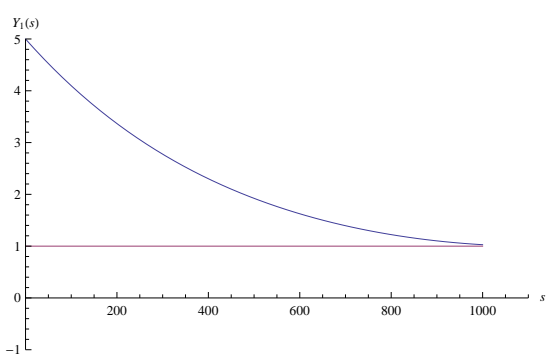

Fig. 4. Numerical solution of Eq. (3.2) for the function $Y_{1}(s)$ obtained in the $Y_{1}-Y_{2}$ plane and with initial conditions $Y_{1}(0)=5, Y_{2}(0)=Y_{3}(0)=Y_{4}(0)=0, Y_{1}^{\prime}(0)=-0.01, Y_{2}^{\prime}(0)=Y_{3}^{\prime}(0)=$ $Y_{4}^{\prime}(0)=0$. The values of parameters are $a=1, m=0.1$ and $v=0.99$. The particle manages to hit the 3 -sphere, which is represented by the horizontal line $Y_{1}=1$.

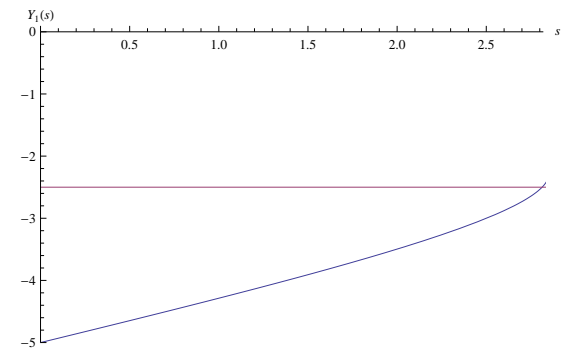

Fig. 5. Numerical solution of Eq. (3.2) for the function $Y_{1}(s)$ obtained in the $Y_{1}-Y_{2}$ plane and with initial conditions $Y_{1}(0)=-5, Y_{2}(0)=Y_{3}(0)=Y_{4}(0)=0, Y_{1}^{\prime}(0)=0.7, Y_{2}^{\prime}(0)=Y_{3}^{\prime}(0)=$ $Y_{4}^{\prime}(0)=0$. The values of parameters are $a=1, m=0.1$ and $v=0.99$. The particle does not manage to hit the 3 -sphere but disappears in correspondence of the $Y_{1}=-2.5$ line.

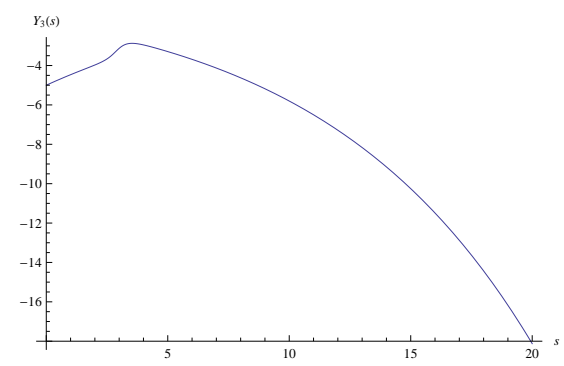

Fig. 6. Numerical solution of Eq. (3.2) for the function $Y_{3}(s)$ obtained in the $Y_{3}-Y_{4}$ plane and with initial conditions $Y_{1}(0)=Y_{2}(0)=0, Y_{3}(0)=Y_{4}(0)=-5, Y_{1}^{\prime}(0)=Y_{2}^{\prime}(0)=0, Y_{3}^{\prime}(0)=$ $Y_{4}^{\prime}(0)=0.566$. The values of parameters are $a=1, m=0.1$ and $v=0.99$. The "antigravity effect" is once again evident. 


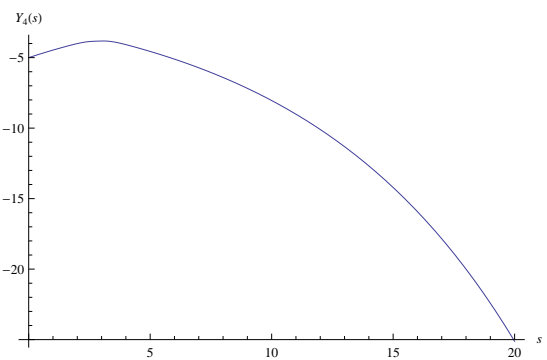

Fig. 7. Numerical solution of Eq. (3.2) for the function $Y_{4}(s)$ obtained in the $Y_{3}-Y_{4}$ plane and with initial conditions $Y_{1}(0)=Y_{2}(0)=0, Y_{3}(0)=Y_{4}(0)=-5, Y_{1}^{\prime}(0)=Y_{2}^{\prime}(0)=0, Y_{3}^{\prime}(0)=$ $Y_{4}^{\prime}(0)=0.566$. The values of parameters are $a=1, m=0.1$ and $v=0.99$. The "antigravity effect" is once again evident.

\section{The coordinate shift method}

A really important issue related to "boosted geometries" is represented by the occurrence of terms quadratic in Dirac's $\delta$ distribution in the Riemann tensor, which makes this object not defined. This Section has two purposes: on one hand it elucidates a new equivalent method for describing the gravitational field of a massless particle (showing that it gives the same results as the ones we have obtained in the previous Sections with the boosting procedure), on the other hand it proposes a recipe for the problem concerning the presence of products of two distributions in the Riemann tensor.

The sources of gravitational (shock-) waves are massless particles moving along a null surface such as a horizon in the case of black holes. Therefore, another way to introduce a gravitational shock-wave is through a coordinate shift which reflects this peculiarity. This method is equivalent to the scissors-and-paste approach introduced by Penrose 29] and can be applied both to vacuum solutions of Einstein equations 27. and in presence of matter fields and non-vanishing cosmological constant [28].

Following Refs. 27/28, we start with the line element

$$
\mathrm{d} s^{2}=2 A(u, v) \mathrm{d} u \mathrm{~d} v+g(u, v) h_{i j}(x) \mathrm{d} x^{i} \mathrm{~d} x^{j},
$$

with $i, j=1,2$ (hereafter $v$ is a spacetime coordinate, unlike the previous Sections where it indicates the boost velocity or the particle velocity). We also assume the presence of some matter fields whose non-vanishing components of the energymomentum tensor are given by

$$
T=2 T_{u v}(u, v, x) \mathrm{d} u \mathrm{~d} v+T_{u u}(u, v, x) \mathrm{d} u^{2}+T_{v v}(u, v, x) \mathrm{d} v^{2}+T_{i j}(u, v, x) \mathrm{d} x^{i} \mathrm{~d} x^{j} .
$$

Consider a massless particle located at $u=0$ and moving with the speed of light in the $v$-direction. The coordinate shift method consists in making the ansatz according to which for $u<0$ the spacetime is still described by (4.1) and for $u>0$ by (4.1) but with $v$ shifted as $v \rightarrow v+f(x)$, where $f(x)$ is a (shift) function to be 
determined. Therefore, the resulting line element reads as

$$
\mathrm{d} s^{2}=2 A(u, v+\Theta f) \mathrm{d} u\left(\mathrm{~d} v+\Theta f_{, i} \mathrm{~d} x^{i}\right)+g(u, v+\Theta f) h_{i j}(x) \mathrm{d} x^{i} \mathrm{~d} x^{j},
$$

where $\Theta=\Theta(u)$ is the Heaviside step function and

$$
\begin{aligned}
T= & 2 T_{u v}(u, v+\Theta f, x) \mathrm{d} u\left(\mathrm{~d} v+\Theta f_{, i} \mathrm{~d} x^{i}\right)+T_{u u}(u, v+\Theta f, x) \mathrm{d} u^{2} \\
& +T_{v v}(u, v+\Theta f, x)\left(\mathrm{d} v+\Theta f_{, i} \mathrm{~d} x^{i}\right)^{2}+T_{i j}(u, v+\Theta f, x) \mathrm{d} x^{i} \mathrm{~d} x^{j} .
\end{aligned}
$$

With the notation

$$
\hat{u}=u, \quad \hat{v}=v+f(x) \Theta(u), \quad \hat{x}^{i}=x^{i},
$$

the metric (4.3) assumes the handy form

$$
\begin{aligned}
\mathrm{d} s^{2} & =2 \hat{A} \mathrm{~d} \hat{u}(\mathrm{~d} \hat{v}-\delta(\hat{u}) \hat{f} \mathrm{~d} \hat{u})+\hat{g} \hat{h}_{i j}(x) \mathrm{d} \hat{x}^{i} \mathrm{~d} \hat{x}^{j} \\
& =2 \hat{A} \mathrm{~d} \hat{u} \mathrm{~d} \hat{v}+\hat{F} \mathrm{~d} \hat{u}^{2}+\hat{g} \hat{h}_{i j}(x) \mathrm{d} \hat{x}^{i} \mathrm{~d} \hat{x}^{j},
\end{aligned}
$$

and the energy-momentum tensor becomes

$T=2\left(\hat{T}_{\hat{u} \hat{v}}-\hat{T}_{\hat{v} \hat{v}} \hat{f} \hat{\delta}\right) \mathrm{d} \hat{u} \mathrm{~d} \hat{v}+\left(\hat{T}_{\hat{u} \hat{u}}+\hat{T}_{\hat{v} \hat{v}} \hat{f}^{2} \hat{\delta}^{2}-2 \hat{T}_{\hat{u} \hat{v}} \hat{f} \hat{\delta}\right) \mathrm{d} \hat{u}^{2}+\hat{T}_{\hat{v} \hat{v}} \mathrm{~d} \hat{v}^{2}+\hat{T}_{i j} \mathrm{~d} \hat{x}^{i} \mathrm{~d} \hat{x}^{j}$,

with $\hat{F}=F(\hat{u}, \hat{v}, \hat{x})=-2 \hat{A} \hat{f} \hat{\delta}$ and where the hats indicate that the corresponding quantities are evaluated at $\hat{u}, \hat{v}, \hat{x}$ and $\hat{\delta}=\delta(\hat{u})$ is the $\delta$ distribution. We now demand that the metric (4.6) satisfies Einstein equation where the energy-momentum tensor is given by Eq. (4.7) plus the the energy-momentum tensor of the massless particle located at the origin of the transverse $x$-space and at $u=0$ and moving at the speed of light in the $v$-direction

$$
T^{p}=T_{u u}^{p} \mathrm{~d} u^{2}=\hat{T}_{u \hat{u}}^{p} \mathrm{~d} \hat{u}^{2}=-4 p \hat{A}^{2} \hat{\delta}^{(2)}(\hat{x}) \hat{\delta}(\hat{u}) \mathrm{d} \hat{u}^{2},
$$

where $p$ is the particle momentum. If we suppose that the parts of field equations that do not involve the function $f$ are automatically satisfied, we find, by examining the terms linear in $f \delta$, that the necessary and sufficient conditions for being able to introduce a gravitational shock-wave via a coordinate shift amount to demand that at $u=0$ there exist the additional conditions (hereafter we drop the hat symbol to simplify the notation)

$$
\begin{gathered}
g_{, v}=A_{, v}=T_{v v}=0, \\
\triangle_{h_{i j}} f-\frac{g_{, u v}}{A} f=32 \pi p g A \delta^{(2)}(x),
\end{gathered}
$$

where

$$
\triangle_{h_{i j}}=\frac{1}{\sqrt{h}} \partial_{i} \sqrt{h} h^{i j} \partial_{j}
$$

is the Laplacian with respect to the 2-metric $h_{i j}$. A crucial point is represented by the presence of $\delta^{2}$ type terms both in Riemann and in Ricci tensors (see Appendix Appendix D. These terms must vanish in a distributional sense, otherwise these 
two tensors are not defined. By considering the conditions (4.9), it is easy to show that the quantities $\frac{A_{, v v}}{A}, \frac{A_{, v}^{2}}{A^{2}}, \frac{g_{, v}}{g}, \frac{A_{, v}}{A}$ appearing both in Riemann and in Ricci tensors are of order $O(u)$ or $O\left(u^{2}\right)$. Since all quantities involving $\delta$ terms should be intended as distributions to be integrated over smooth functions, we can conclude that all these $\delta^{2}$ terms give vanishing contribution and therefore both Riemann and Ricci tensors turn out to be under control as functions (in a distributional sense) of spacetime coordinates $\left\{u, v, x^{1}, x^{2}\right\}$, as advocated in Ref. 28. The geodesic equations for the metric (4.6) obtained by varying the coordinates $v$ and $x^{i}$ are (dots denote derivatives with respect to the affine parameter)

$$
\begin{gathered}
\ddot{u}+\frac{A_{, u}}{A} \dot{u}^{2}-\frac{g, v}{2 A} h_{i j} \dot{x}^{i} \dot{x}^{j}+f \frac{A_{, v}}{A} \delta \dot{u}^{2}=0, \\
\ddot{x}^{i}+\Gamma_{j k}^{i} \dot{x}^{j} \dot{x}^{k}+\frac{g_{, u}}{g} \dot{u} \dot{x}^{i}+\frac{g, v}{g} \dot{v} \dot{x}^{i}+\frac{A}{g} \delta f_{, i} h^{i j} \dot{u}^{2}=0,
\end{gathered}
$$

where $\Gamma_{j k}^{i}$ denote the Christoffel symbols (see Appendix A of Ref. [28] for their lengthy expression); the geodesic equation obtained from the variation of $u$ is

$$
\begin{aligned}
& \ddot{v}+\frac{A_{, v}}{A} \dot{v}^{2}-\frac{g_{, u}}{2 A} h_{i j} \dot{x}^{i} \dot{x}^{j}+\left(f \frac{A_{, u}}{A} \dot{u}^{2}-2 f \frac{A_{, v}}{A} \dot{u} \dot{v}-2 f_{, i} \dot{u} \dot{x}^{i}-\frac{g_{, v}}{A} f h_{i j} \dot{x}^{i} \dot{x}^{j}\right) \delta \\
& -f \delta^{\prime} \dot{u}^{2}+2 f^{2} \delta^{2} \frac{A_{, v}}{A} \dot{u}^{2}=0 .
\end{aligned}
$$

On performing the integration of the geodesic equations, it is possible to understand how the original geometry (4.1) is affected by the presence of a massless particle moving in the $v$-direction at $u=0$. In fact, as the geodesic trajectory crosses the null surface $u=0$ there is a shift in its $v$-component expressed by the relation

$$
\left.\Delta v \equiv v\right|_{u=0^{+}}-\left.v\right|_{u=0^{-}}=f(x),
$$

and a refraction effect in the transverse $x$-plane expressed by the refraction function

$$
\left.R^{i}(x) \equiv \frac{\mathrm{d} x^{i}}{\mathrm{~d} u}\right|_{u=0^{-}}-\left.\frac{\mathrm{d} x^{i}}{\mathrm{~d} u}\right|_{u=0^{+}}=\left.\frac{A}{g}\right|_{u=0} f_{, i} h^{i j},
$$

which measures the change of the angle that the trajectory forms with the $u=0$ surface after having crossed it. Therefore, when a trajectory crosses the $u=0$ null surface its $v$ component suffers from a discontinuity which, according to (4.15), equals $f(x)$, while the other components remain continuous. Moreover, Eq. (4.16) expresses the fact that the directional derivatives of $f(x)$ give information about how much the $x^{i}$ components change direction along $u$ while crossing the surface $u=0$.

The examples analyzed in Ref. 28] show that, in order to bring the metric (1.12) in the form (4.1), we should introduce the function

$$
F: r \rightarrow F(r)=\exp \left[\frac{1}{a} \int \mathrm{d} r \frac{r a^{2}}{\left(r a^{2}-r^{3}-2 m a^{2}\right)}\right],
$$


and the new independent variables

$$
u=\mathrm{e}^{t / a} F(r), \quad v=\mathrm{e}^{-t / a} F(r) .
$$

Bearing in mind these relation and Eq. (4.1), we have that

$$
A(u, v)=\frac{\left(1-\frac{2 m}{r}-\frac{r^{2}}{a^{2}}\right) a^{2}}{2} F^{-2},
$$

By performing the integration, we have found that

$F(r)=\exp \left(a \frac{r_{1}\left(r_{3}-r_{2}\right) \log \left(r-r_{1}\right)+r_{2}\left(r_{1}-r_{3}\right) \log \left(r-r_{2}\right)+r_{3}\left(r_{2}-r_{1}\right) \log \left(r-r_{3}\right)}{\left(r_{1}-r_{2}\right)\left(r_{1}-r_{3}\right)\left(r_{2}-r_{3}\right)}\right)$,

where $r_{1}, r_{2}$ and $r_{3}$ are the three roots of the cubic equation

$$
r^{3}-r a^{2}+2 m a^{2}=0,
$$

whose value is given by

$$
\begin{gathered}
r_{1}=\frac{1}{3^{1 / 3}}\left(\frac{a^{2}}{\Upsilon}+\frac{\Upsilon}{3^{1 / 3}}\right), \\
r_{2,3}=\frac{1}{2} \frac{1}{3^{1 / 3}}\left(-\frac{(1 \pm i \sqrt{3}) a^{2}}{\Upsilon}-\frac{(1 \mp i \sqrt{3}) \Upsilon}{3^{1 / 3}}\right),
\end{gathered}
$$

where $\Upsilon$ is defined as

$$
\Upsilon \equiv\left(-9 a^{2} m+\sqrt{3} \sqrt{27 a^{4} m^{2}-a^{6}}\right)^{1 / 3} .
$$

In other words, Eqs. (4.23)-(4.24) describe the three (null) surfaces where the metric (1.12) blows up, and hence the three horizons that characterize this geometry. With the hypothesis $a / m>\sqrt{27}$ (which is respected by the choice $a=1$ and $m=0.1$ adopted in the last Section) the discriminant of (4.22) becomes negative and then (4.23)-(4.24) turn out to be real roots. This condition allows us to write the roots (4.23) -4.24) in trigonometric form. We obtain

$$
\begin{gathered}
r_{1}=\frac{2 a}{\sqrt{3}} \cos \left(\frac{\varphi}{3}\right), \\
r_{2,3}=-\frac{2 a}{\sqrt{3}} \cos \left(\frac{\varphi \mp \pi}{3}\right)=-\frac{a}{\sqrt{3}}\left(\cos \frac{\varphi}{3} \pm \sqrt{3} \sin \frac{\varphi}{3}\right),
\end{gathered}
$$

where $\cos \varphi=\sqrt{27} \mathrm{~m} / \mathrm{a}$. Note also that the roots (4.23)-(4.24) are characterized by the fact that $r_{1}+r_{2}+r_{3}=0$ and $r_{1} r_{2} r_{3}=-2 m a^{2}$. Now we can write (4.21) as

$$
F(r)=\prod_{i=1}^{3}\left(r-r_{i}\right)^{k_{i}}
$$


where the three constants $k_{i}(i=1, \ldots, 3)$ are given by

$$
\begin{aligned}
& k_{1}=\frac{a r_{1}\left(r_{3}-r_{2}\right)}{k_{r}}, \\
& k_{2}=\frac{a r_{2}\left(r_{1}-r_{3}\right)}{k_{r}}, \\
& k_{3}=\frac{a r_{3}\left(r_{2}-r_{1}\right)}{k_{r}},
\end{aligned}
$$

with $k_{r}=\left(r_{1}-r_{2}\right)\left(r_{1}-r_{3}\right)\left(r_{2}-r_{3}\right)$. Therefore, bearing in mind (4.18) and (4.19) we have that

$$
\begin{gathered}
A(u, v)=-\frac{1}{2 r} \prod_{i=1}^{3}\left(r-r_{i}\right)^{1-2 k_{i}}, \\
u=\mathrm{e}^{t / a} \prod_{i=1}^{3}\left(r-r_{i}\right)^{k_{i}}, \\
v=\mathrm{e}^{-t / a} \prod_{i=1}^{3}\left(r-r_{i}\right)^{k_{i}}
\end{gathered}
$$

and in particular we can satisfy the condition $u=0$ for $r=r_{i}(i=1, \ldots, 3)$. Next, before writing down the equation satisfied by the shift function $f(\theta)$, we have to show that conditions (4.9) are satisfied. Having obtained the following relations for the derivatives:

$$
\begin{aligned}
& \frac{\mathrm{d}}{\mathrm{d} u}=\frac{1}{2}\left(\frac{a \mathrm{e}^{-t / a}}{F(r)} \frac{\mathrm{d}}{\mathrm{d} t}+\frac{\mathrm{e}^{-t / a}}{F^{\prime}(r)} \frac{\mathrm{d}}{\mathrm{d} r}\right), \\
& \frac{\mathrm{d}}{\mathrm{d} v}=\frac{1}{2}\left(\frac{-a \mathrm{e}^{t / a}}{F(r)} \frac{\mathrm{d}}{\mathrm{d} t}+\frac{\mathrm{e}^{t / a}}{F^{\prime}(r)} \frac{\mathrm{d}}{\mathrm{d} r}\right),
\end{aligned}
$$

we find that

$$
g_{, v}=\mathrm{e}^{\frac{t}{a}} \frac{r\left(r-r_{1}\right)^{1-k_{1}}\left(r-r_{2}\right)^{1-k_{2}}\left(r-r_{3}\right)^{1-k_{3}}}{k_{1}\left(r-r_{2}\right)\left(r-r_{3}\right)+k_{2}\left(r-r_{1}\right)\left(r-r_{3}\right)+k_{3}\left(r-r_{1}\right)\left(r-r_{2}\right)},
$$

and therefore

$$
\lim _{u \rightarrow 0} g_{, v}=0 \quad \text { iff } k_{i}<1 .
$$

Moreover,

$$
A_{, v}=\mathrm{e}^{\frac{t}{a}} \frac{\left(r-r_{1}\right)^{1-3 k_{1}}\left(r-r_{2}\right)^{1-3 k_{2}}\left(r-r_{3}\right)^{1-3 k_{3}}}{4 r^{2}\left[k_{1}\left(r-r_{2}\right)\left(r-r_{3}\right)+\left(r-r_{1}\right)\left(k_{2}\left(r-r_{3}\right)+k_{3}\left(r-r_{2}\right)\right]^{2}\right.} \mathcal{F},
$$


where $\mathcal{F}=\mathcal{F}\left(r, r_{i}, k_{i}\right)$ is a function of $r$, the roots (4.23)-(4.24) and the constants $k_{i}$ (which tend to a constant when $r \rightarrow r_{i}$ ), whose particular form is not of any special interest now. We can conclude that

$$
\lim _{u \rightarrow 0} A_{, v}=0 \quad \text { iff } k_{i}<1 / 3 .
$$

By virtue of (4.38) and (4.40) we can say that conditions (4.9) are satisfied provided that

$$
k_{i}<1 / 3, \quad(i=1, \ldots, 3) .
$$

By applying the shift coordinate method to the metric (1.12), we obtain the desired shock wave geometry and we find that the partial differential equation (4.10) satisfied by the shift function $f(\theta)$ becomes

$$
\triangle_{(2)} f-c f=2 \pi k \delta(\xi-1) \delta(\phi),
$$

with

$$
\triangle_{(2)}=\partial_{\xi}\left(1-\xi^{2}\right) \partial_{\xi}+\frac{\partial_{\phi}^{2}}{\left(1-\xi^{2}\right)}, \quad \text { with } \quad \xi=\cos \theta
$$

being the Laplacian on the unit 2-sphere having metric $\mathrm{d} s^{2}=\mathrm{d} \theta^{2}+\sin ^{2} \theta \mathrm{d} \phi^{2}$, and $k$ and $c$ being real constants. This equation represents the usual Legendre equation of order $n$ ( $n$ being a solution of $n(n+1)+c=0)$ with a Dirac's $\delta$ appearing on the right-hand side. Therefore, its solutions depend strongly on the values assumed by the constant $c$ and can be given in terms of Legendre polynomials as

$$
f(\theta ; c)=-k \sum_{l=0}^{+\infty} \frac{\left(l+\frac{1}{2}\right)}{[l(l+1)+c]} P_{l}(\cos \theta), \quad c \in \mathbb{R}-\{-N(N+1), N=0,1, \ldots\} .
$$

In the case of Schwarzschild-de Sitter black hole, (4.42) depends on the ratio $a / m$ and thus possesses two branches of solutions for the constants $c$ and $k$. In the branch where the null surface is described by a positive value of $r$ we have that

$$
c=\frac{\left(r_{1}-r_{3}\right)\left(r_{3}-r_{2}\right)}{a^{2}}=2 \sin \left(\frac{\varphi}{3}\right)\left[\sqrt{3} \cos \left(\frac{\varphi}{3}\right)-\sin \left(\frac{\varphi}{3}\right)\right],
$$

while the constant $k$ is always positive, with precise value which is not of particular interest. The inequality $a / m>\sqrt{27}$ is equivalent to the obvious condition $\cos \varphi<$ 1 , moreover the null hypersurface $u=0$ where the massless particle is placed corresponds to $r=r_{3}$ (see Eq. (4.27)). The condition $r_{3}>0$ implies that (for positive values of $m$ and $a) \varphi \in\left(\pi / 2, \frac{3}{2} \pi\right]$, so that

$$
c \in(-2,0) \cup(0,1) \quad \text { if } \quad \varphi \in(\pi / 2, \pi) \cup\left(\pi, \frac{3}{2} \pi\right) .
$$

The boundary cases $c=-2\left(\varphi=\frac{3}{2} \pi\right)$ and $c=1(\varphi=\pi / 2)$ correspond to de Sitter spacetime and Schwarzschild black hole, respectively, whereas the case $c=0$ 
$(\varphi=\pi)$ is similar to the extremal Reissner-Nordström charged black hole. The shift function $f(\theta)$ is given by Eq. (4.44). For $\frac{1}{4} \leq c<1$, an integral representation of the solution is given by 28 ]

$$
\begin{aligned}
f(\theta ; c) & =\frac{-k}{\sqrt{2}} \int_{0}^{+\infty} \mathrm{d} s \cos (\sqrt{c-1 / 4} s) \frac{1}{\sqrt{\cosh s-\cos \theta}} \\
& =\frac{-k \pi}{2 \cosh (\sqrt{c-1 / 4} \pi)} F\left(1 / 2-i \sqrt{c-1 / 4}, 1 / 2+i \sqrt{c-1 / 4} ; 1 ; \cos ^{2} \frac{\theta}{2}\right),
\end{aligned}
$$

where $F(a, b ; c ; z)$ is the Gaussian or ordinary hypergeometric function. For $0<c \leq$ $\frac{1}{4}$ the solution is given by replacing $\sqrt{c-1 / 4}$ by $i \sqrt{1 / 4-c}$ and the trigonometric functions by hyperbolic ones, and vice versa. In both cases the shift function blows up at the point of the unit 2-sphere where the particle is located, i.e. at the northern pole $\theta=0$. Moreover, it is everywhere negative and for fixed $c$ it is a monotonically increasing function of $\theta \in[0, \pi]$, approaching a nonvanishing constant at $\theta=\pi$. For fixed $\theta$ it also monotonically increases as a function of $c \in(0,1)$. The refraction function (4.16) is given by

$$
R(\theta ; c)=\left(\frac{A}{g}\right)_{u=0} \partial_{\theta} f(\theta ; c) .
$$

It is a monotonically decreasing function of $\theta$ such that $\lim _{\theta \rightarrow 0} R(\theta ; c)=+\infty$ and $\lim _{\theta \rightarrow \pi} R(\theta ; c)=0$. Thus, both the shift function and the refraction function blow up at $\theta=0$ and reach their minimum magnitudes at the southern pole $\theta=\pi$, where the refraction phenomenon disappears even if a particle trajectory is still discontinuous since $f(\pi ; c) \neq 0$. For $-2<c<0$, the shift function is given by the integral representation

$$
f(\theta ; c)=\frac{-k}{2 c}-k \int_{0}^{+\infty} \mathrm{d} s \cosh (\sqrt{1 / 4-c} s)\left(\frac{1 / \sqrt{2}}{\sqrt{\cosh s-\cos \theta}}-\mathrm{e}^{-s / 2}\right) .
$$

The solution again blows up at $\theta=0$ and it monotonically increases as we move from $\theta=0$ to $\theta=\pi$. Moreover, it changes from negative to positive values at an angle $\theta_{0}$ that depends on the value assumed by the constant $c$ and reaches its minimum at $\theta=0$. On the other hand, the refraction function is a monotonically decreasing function of $\theta$.

As we can see, the conditions found in this Section via the coordinate shift method are not in contrast with the results obtained through the boosting procedure of the previous Sections. We have shown in fact that the "boosted horizon" gives rise to a sort of "antigravity effect" which, in light of the results displayed in this Section, can be read as the refraction phenomenon described by the function (4.16). It is an important result the fact these effects take place in nonsingular region of 
spacetime, i.e. the "boosted horizon" (for the boosting picture) and at the null hypersurface $u=0$ (in the coordinate shift method). Moreover, the presence of the singularity 3 -sphere where the Kretschmann invariant is not defined could be probably related to the discontinuity of the $v$ component defined by Eq. (4.15). The fact that in the ultrarelativistic regime the "boosted horizon" and the singularity 3 -sphere positions' get blurred (as shown in Sec. III) represents a clue in favour of this hypothesis. To make more clear the equivalence between the boost and the coordinate shift method, one should be able to relate the $\left\{u, v, x^{1}, x^{2}\right\}$ coordinates of the metric (4.6) with the $\left\{Y_{1}, Y_{2}, Y_{3}, Y_{4}\right\}$ coordinates of the four-dimensional metric components (2.21)-2.30). This can be done with the help of the results of Appendix Appendix E. Therefore, by exploiting the equivalence between the two methods and the relations relating the two sets of coordinates, it is possible to relate all the results obtained through the coordinate shift method to those achieved with the boosting procedure. This means that also the considerations about how handling the $\delta^{2}$ terms in the Riemann tensor are valid also if we use the boost picture. Thus, the severe singularities of the Riemann tensor associated with metric (1.13) can be considered to be under control.

\section{Concluding remarks and open problems}

We have numerically evaluated, for the first time in the literature, the Riemann curvature of a boosted spacetime in the ultrarelativistic limit $v \rightarrow 1$, starting from Schwarzschild-de Sitter spacetime metric (1.12). We have exploited the fact that a de Sitter spacetime can be seen as a four-dimensional hyperboloid embedded in a flat five-dimensional spacetime and satisfying the constraint (2.2). After that, we have introduced the boosting procedure through the relations (2.15)-(2.17) which make it possible to obtain the boosted Schwarzschild-de Sitter metric (2.18), whose ultrarelativistic limit is represented by (1.13). By exploiting the hyperboloid constraint (2.2) we have then expressed (2.18) in the manifestly four-dimensional form (2.21)-(2.30). By virtue of (2.2), the metric components (2.21)-(2.30) are defined only if $\sigma>0, \sigma$ being defined by relation (2.19). This fact is strictly related to inequality (3.3). In fact, $\left\{\partial / \partial Y_{\mu}\right\}$ being a coordinate basis, we have numerically computed the Riemann curvature tensor by using the usual relations of general relativity, and to better understand the features of curvature we have studied both the Kretschmann invariant and the geodesic equation (3.2). We have indeed found that the Kretschmann invariant is not defined unless (3.3) holds and thus we have just concluded that there exists a 3 -sphere of radius $a$ where the spacetime possesses a "scalar curvature singularity". In fact, from the numerical analysis of the geodesic equation, we have found that if the particle lies initially on the positive half-line $Y_{1}>0, Y_{2}=0$ of Fig. 1 it always reaches the 3 -sphere (Fig. 4). After that, its geodesic is no longer defined and hence we can conclude that the 3 -sphere of equation $\left(Y_{1}\right)^{2}+\left(Y_{2}\right)^{2}+\left(Y_{3}\right)^{2}+\left(Y_{4}\right)^{2}=a^{2}$ defines a "scalar curvature singularity" for the "boosted geometry" under investigation. When the particle lies initially on 
the negative half-line $Y_{1}<0, Y_{2}=0$, its geodesic is not defined even before it manages to reach the 3 -sphere (see Fig. 5): there exists a "scalar curvature singularity" whose position depends on the boost velocity $v$.

We have also discovered that "boosted geometries" are characterized by the presence of a sort of elastic wall surrounding the 3 -sphere whose coordinates depend only on the boost velocity (see Tab.11). All geodesics indeed, despite being complete, are always pushed away from there, as Figs. 2 and 3 show. We propose to call this barrier "boosted horizon" because, as in the case of Schwarzschild geometry, it is not a singularity of spacetime, but it is related to a sort of "antigravity effect" that should rule "boosted geometries".

As we know, boosted geometries are ruled by the fact that both the spacetime metric and the Riemann curvature tensor assume a distributional nature in the ultrarelativistic regime. This regime is still ruled by "antigravity effects", with the peculiarity that "boosted horizon" and singularity 3-sphere tend to overlap.

In the last part of the paper we have analyzed the geometry of the metric (1.12) through the coordinate shift method. We have shown that this new picture is equivalent to the boosting procedure and we have demonstrated how it solves the problem of the presence of $\delta^{2}$ terms in the Riemann tensor. In particular, the "antigravity effects" emerged at the "boosted horizon" have been ascribed to the refraction phenomenon described by the function (4.16). Moreover, the fact that in the ultrarelativistic regime the "boosted horizon" position's tends to that of the singularity 3 -sphere could be related to the fact that, in the coordinate shift method picture, when the particle crosses the null surface located at $u=0$ it suffers a discontinuity in its $v$-component (Eq. (4.15)) while the $x^{i}$ components are refracted according to (4.16). This is a really delicate point as, unlike the singularity 3 -sphere, both the null hypersurface $u=0$ (coordinate shift method) and our "boosted horizon" (boosted picture) do not define a spacetime singularity, and we feel that some more efforts should be produced in this direction. The equivalence between the two methods (demonstrated in Ref. [27]), which can be formally made manifest for our "boosted geometry" by the results of Appendix Appendix E has enabled us to conclude that the Riemann tensor associated with metric (1.13) is defined and has a behavior under control.

We suppose that "antigravity effects" may result from the term $\Lambda=3 / a^{2}>$ 0 occurring in the Schwarzschild-de Sitter metric (1.12) (a positive cosmological constant $\Lambda$ represents a repulsive interaction), while "scalar curvature singularities" might be related to the presence of a more exotic object, i.e. a firewall 3839 , 40, which can be a possible solution to an apparent inconsistency in black hole complementarity 4142 .

\section{acknowledgments}

B.E. is grateful to Marcello Ortaggio for conversations. G. E. is grateful to the Dipartimento di Fisica of Federico II University, Naples, for hospitality and support. 


\section{Appendix A. The Riemann curvature tensor}

Since our paper is addressed to a wide physics audience, we recall here some basic properties of pseudo-Riemannian geometry. The Riemann tensor can be defined in various alternative (and equivalent) ways [30, 43]. First, it can be defined as the map $R: \mathcal{X}(M) \otimes \mathcal{X}(M) \otimes \mathcal{X}(M) \rightarrow \mathcal{X}(M)(\mathcal{X}(M)$ being the set of all vector fields defined on the manifold $M)$ such that

$$
R(X, Y, Z) \equiv \nabla_{X} \nabla_{Y} Z-\nabla_{Y} \nabla_{X} Z-\nabla_{[X, Y]} Z .
$$

In the case in which $[X, Y]=0$, the previous formula reduces to

$$
R(X, Y, Z) \equiv \nabla_{X} \nabla_{Y} Z-\nabla_{Y} \nabla_{X} Z .
$$

Therefore, we can say that the Riemann tensor measures the failure of successive operations of differentiation to commute when applied to a dual vector field (which can be interpreted as the integrability obstruction for the existence of an isometry with Euclidean space), that is (in abstract index notation)

$$
\nabla_{a} \nabla_{b} \omega_{c}-\nabla_{b} \nabla_{a} \omega_{c}=-R_{c a b}^{d} \omega_{d}
$$

Moreover, we can say that the failure of a vector to return to its original value when parallel transported around a small closed loop is directly connected to the Riemann tensor, which is in this way related to the path dependence of parallel transport. We can easily construct a small closed loop at $p \in M$ by choosing a twodimensional surface $S$ through $p$ and choosing coordinates $t$ and $s$ in the surface. Next we construct the loop by moving of a quantity $\Delta t$ along the $s=0$ curve, followed by moving $\Delta s$ along the $t=\Delta t$ curve and then we revert by $\Delta t$ and $\Delta s$. If we consider the vector $v^{a}$ at $p$ and parallel trasport it around the closed loop we have just constructed, the change $\delta v^{a}$ to second order in the displacement $\Delta t$, $\Delta s$ that we register when we revert to the starting point involves once again the Riemann tensor, because we have

$$
\delta v^{a}=\Delta t \Delta s v^{d} T^{c} S^{b} R_{d c b}^{a},
$$

where $T^{c}, S^{b}$ indicate the tangent to the curves of constant $s$ and $t$, respectively. Finally, the Riemann tensor appears also in the geodesic deviation equation, the equation which measures the tendency of geodesics to accelerate toward or away from each other. If $\gamma_{s}(t)$ denotes a smooth 1-parameter family of geodesics such that for each $s \in \mathbb{R}$ the curve $\gamma_{s}$ is a geodesic with affine parameter $t$, the geodesic deviation equation reads as

$$
a^{c} \equiv T^{a} \nabla_{a}\left(T^{b} \nabla_{b} X^{c}\right)=R_{d e f}^{c} T^{d} T^{e} X^{f},
$$

where $a^{c}$ is the relative acceleration of an infinitesimally nearby geodesic in the family, $X^{a}=\partial x^{a}(s, t) / \partial s$ is the deviation vector $\left(x^{a}(s, t)\right.$ being the coordinates of one geodesic of the family $\left.\gamma_{s}(t)\right)$ and $T^{b}=\partial x^{a}(s, t) / \partial t$ represents the vector tangent to the geodesic. Therefore the equation (A.5) states that, if the curvature does not vanish, some initially parallel geodesics will fail to remain parallel: in the 
presence of a gravitational field the fifth postulate of Euclidean geometry is no longer valid.

\section{Appendix B. The tetrad formalism}

In most situations a curvature calculation that relies upon Christoffel symbols is extremely lengthy and not obviously feasible or readable. However, the tetrad formalism is known to simplify such a task, at least when the metric does not possess distributional singularities. Thus, this appendix is devoted to some effort we made to express the highly singular ultrarelativistic boosted metric (1.13) in terms of tetrads.

As in the case of the boosted metric (2.18), starting from the ultrarelativistic metric (1.13) we can arrive at its manifestly four-dimensional form by exploiting (2.19) and (2.20) and hence we can eventually write the covariant metric components in the concise form

$$
\begin{gathered}
g_{k k}=1-\frac{Y_{k}^{2}}{\sigma\left(Y_{j}\right)}+\left(\frac{Y_{k}^{2}}{\sigma\left(Y_{j}\right)}+\delta_{1 k}\right) f\left(Y_{4}\right) \delta\left(Y_{1}+\sqrt{\sigma\left(Y_{j}\right)}\right), \forall k=1,2,3,4, \\
g_{1 k}=-\frac{Y_{1} Y_{k}}{\sigma\left(Y_{j}\right)}+\left(\frac{Y_{1}}{\sqrt{\sigma\left(Y_{j}\right)}}+1\right) \frac{Y_{k}}{\sqrt{\sigma\left(Y_{j}\right)}} f\left(Y_{4}\right) \delta\left(Y_{1}+\sqrt{\sigma\left(Y_{j}\right)}\right), \forall k=2,3,4, \\
g_{2 k}=-\frac{Y_{2} Y_{k}}{\sigma\left(Y_{j}\right)}+\frac{Y_{2} Y_{k}}{\sigma\left(Y_{j}\right)} f\left(Y_{4}\right) \delta\left(Y_{1}+\sqrt{\sigma\left(Y_{j}\right)}\right), \forall k=3,4, \\
g_{34}=-\frac{Y_{3} Y_{4}}{\sigma\left(Y_{j}\right)}+\frac{Y_{3} Y_{4}}{\sigma\left(Y_{j}\right)} f\left(Y_{4}\right) \delta\left(Y_{1}+\sqrt{\sigma\left(Y_{j}\right)}\right)
\end{gathered}
$$

where

$$
f\left(Y_{4}\right) \equiv 4 p\left[-2+\frac{Y_{4}}{a} \log \left(\frac{a+Y_{4}}{a-Y_{4}}\right)\right] .
$$

Since all components of this metric are nonvanishing, at this stage we still assume the existence of tetrad covectors $e^{a}{ }_{\mu}$ such that the covariant form of the metric reads as

$$
g_{\mu \nu}=e_{\mu}^{a} e^{b}{ }_{\nu} \eta_{a b},
$$

$a, b$ being Lorentz-frame indices, and $\eta_{a b}$ being the familiar Minkowski metric $\operatorname{diag}(-1,1,1,1)$. By comparison of the formulae (B.1)-(B.4) with (B.6) we find that one can set

$$
e_{k}^{0}=\frac{Y_{k}}{\sqrt{\sigma\left(Y_{j}\right)}}, \forall k=1,2,3,4
$$


while the other components of the singular, distribution-valued limit of tetrad covectors solve the following nonlinear algebraic system:

$$
\begin{gathered}
\left(e_{k}^{1}\right)^{2}+\left(e_{k}^{2}\right)^{2}+\left(e_{k}^{3}\right)^{2}=1+\left(\frac{Y_{k}^{2}}{\sigma\left(Y_{j}\right)}+\delta_{1 k}\right) f\left(Y_{4}\right) \delta\left(Y_{1}+\sqrt{\sigma\left(Y_{j}\right)}\right), \forall k=1,2,3,4 \\
\sum_{i=1}^{3} e_{1}^{i} e^{i}=\left(\frac{Y_{1}}{\sqrt{\sigma\left(Y_{j}\right)}}+1\right) \frac{Y_{k}}{\sqrt{\sigma\left(Y_{j}\right)}} f\left(Y_{4}\right) \delta\left(Y_{1}+\sqrt{\sigma\left(Y_{j}\right)}\right), \forall k=2,3,4, \quad \text { (B.9) } \\
\sum_{i=1}^{3} e_{2}^{i} e^{i}{ }_{k}=\frac{Y_{2} Y_{k}}{\sigma\left(Y_{j}\right)} f\left(Y_{4}\right) \delta\left(Y_{1}+\sqrt{\sigma\left(Y_{j}\right)}\right), \forall k=3,4 \\
\sum_{i=1}^{3} e_{3}^{i} e^{i}{ }_{4}=\frac{Y_{3} Y_{4}}{\sigma\left(Y_{j}\right)} f\left(Y_{4}\right) \delta\left(Y_{1}+\sqrt{\sigma\left(Y_{j}\right)}\right)
\end{gathered}
$$

Since the system (B.8)-(B.11) consists of 10 equations for the 12 unknown tetrad covectors, it is possible to find at least a particular solution. Now, once we get such a solution, the procedure should be as follows. As we know from general relativity, whenever the spacetime manifold is parallelizable, we can always introduce a set of Lorentz frames [44, so that the spin-connection 1-form $\omega^{a b}=\omega_{\mu}^{a b} d x^{\mu}$ obtained from requiring that the torsion 2-form should vanish has components 45]

$$
\omega_{\mu}^{a b}=\frac{1}{2} e^{a \nu}\left(e_{\nu, \mu}^{b}-e_{\mu, \nu}^{b}\right)-\frac{1}{2} e^{b \nu}\left(e_{\nu, \mu}^{a}-e_{\mu, \nu}^{a}\right)+\frac{1}{2} e^{a \nu} e^{b \sigma}\left(e_{\nu, \sigma}^{c}-e_{\sigma, \nu}^{c}\right) e_{c \mu},
$$

where

$$
e^{a \nu}=\eta^{a b} e_{b}^{\nu}, e_{c \mu}=e_{\mu}^{a} \eta_{a c},
$$

the tetrad vectors $e_{a}^{\mu}$ being computable by comparison from the relation

$$
\mathrm{d} x^{\mu}=e_{a}^{\mu} e^{a},
$$

which holds by virtue of the definition of tetrad 1-forms

$$
e^{a} \equiv e_{\mu}^{a} d x^{\mu},
$$

jointly with 45

$$
e_{a}^{\rho} e_{\mu}^{a}=\delta_{\mu}^{\rho} .
$$

At this stage, we should be able to perform the curvature calculation bearing in mind that the Riemann curvature is described by the 2 -form

$$
R^{a b}=\frac{1}{2} R_{\mu \nu}^{a b} d x^{\mu} \wedge d x^{\nu},
$$

where the components are given by

$$
R_{\mu \nu}^{a b}=\left(\omega_{\nu, \mu}^{a b}-\omega_{\mu, \nu}^{a b}\right)+\eta_{c d}\left(\omega_{\mu}^{b d} \omega_{\nu}^{c a}-\omega_{\mu}^{a d} \omega_{\nu}^{c b}\right)
$$


By virtue of Secs. II and III, the singular limit of the curvature 2-form is a nontrivial mathematical object, since it involves the Dirac delta distribution, its fractional powers and its derivatives. Finally, the Riemann curvature tensor $R_{\nu \rho \sigma}^{\mu}$ can be obtained from the identity

$$
R_{\nu \rho \sigma}^{\mu} e_{\mu}^{a}=R_{b \rho \sigma}^{a} e_{\nu}^{b} .
$$

\section{Appendix C. The b-completeness of spacetime}

The b-boundary construction is a device to attach to any spacetime a set of boundary points. Such a boundary point can be considered as an equivalence class of inextendible curves in a spacetime, whose affine length is finite 31 35.

Let $\lambda(t)$ be a $C^{1}$ curve through a point $p$ of a manifold $M$ and let $\left\{E_{\mu}\right\}$ (as before $\mu=1,2,3,4)$ be a basis for the tangent vector space at $p$ to the manifold, $T_{p} M$. We can propagate $\left\{E_{\mu}\right\}$ along $\lambda(t)$ to obtain a basis for $T_{\lambda(t)} M, \forall t$. Then any $V=(\partial / \partial t)_{\lambda(t)} \in T_{\lambda(t)} M$ can be expressed as $V=V^{\mu}(t) E_{\mu}$ and we can define a generalized affine parameter $u$ on the curve $\lambda(t)$ by

$$
u=\int_{p}\left(\sum_{\mu} V_{\mu} V^{\mu}\right)^{1 / 2} \mathrm{~d} t .
$$

Let $\left\{E_{\mu^{\prime}}\right\}$ be another basis of $T_{p} M$. Then there exists some nonsingular matrix $A^{\mu}{ }_{\nu}$ such that

$$
E_{\nu}=\sum_{\mu^{\prime}} A_{\nu}^{\mu^{\prime}} E_{\mu^{\prime}}
$$

As $\left\{E_{\mu^{\prime}}\right\}$ and $\left\{E_{\mu}\right\}$ are parallely transported along $\lambda(t)$, this relation is valid with constant $A^{\mu}{ }_{\nu}$ and hence we have

$$
V^{\mu^{\prime}}(t)=\sum_{\nu} A_{\nu}^{\mu^{\prime}} V^{\nu}(t)
$$

Since $A^{\mu}{ }_{\nu}$ is nonsingular, there exists some constant $C>0$ such that

$$
C \sum_{\mu} V_{\mu} V^{\mu} \leq \sum_{\mu^{\prime}} V_{\mu^{\prime}} V^{\mu^{\prime}} \leq C^{-1} \sum_{\mu} V_{\mu} V^{\mu} .
$$

Thus, the length of a curve $\lambda$ is finite in the parameter $u$ if and only if it is finite in the parameter $u^{\prime}$. If $\lambda$ is a geodesic then $u$ becomes its affine parameter, but the definition given above is still valid since it has been formulated in terms of a general parameter $u$ defined on any $C^{1}$ curve. Therefore, we say that a spacetime $(M, g)$ is b-complete if there exists an endpoint for every $C^{1}$ curve of finite length as measured by a generalized affine parameter. We have that b-completeness implies g-completeness (short for geodesic completeness), but the converse is not true. Therefore, we can define a spacetime to be singularity-free if it is b-complete. This means that g-completeness represents the minimum condition for a spacetime to be considered singularity-free. 


\section{Appendix D. $\delta^{2}$ terms in Riemann and Ricci tensors}

We find that the only Riemann tensor components of the metric (4.6) depending on $\hat{\delta}^{2}=\delta^{2}(\hat{u})$ are given by (we drop the hat symbol to ease the notation)

$$
\begin{gathered}
R_{u v u}^{v}=2\left(A_{, u v}-\frac{A_{, u} A_{, v}}{A}\right) f \delta+2\left(\frac{A_{, v v}}{A}-\frac{A_{, v}^{2}}{A^{2}}\right) f^{2} \delta^{2}, \\
R_{u x^{i} u}^{v}=\left(2 \frac{A_{, v}}{A}-\frac{g_{, v}}{g}\right) f_{, x^{i}} f \delta^{2}, \quad(i=1,2), \\
R_{u x^{i} u}^{x^{i}}=\left(\frac{g_{, v}}{g} \frac{A_{, v}}{A}\right) f^{2} \delta^{2}+\ldots(\text { terms at most linear in } \delta), \quad(i=1,2) .
\end{gathered}
$$

Therefore the only Ricci tensor component having $\delta^{2}$ terms is

$$
\begin{aligned}
R_{u u} & =\sum_{\rho} R_{u \rho u}^{\rho}=R_{u v u}^{v}+R_{u x^{1} u}^{x^{1}}+R_{u x^{2} u}^{x^{2}} \\
& =2\left(\frac{A_{, v v}}{A}-\frac{A_{, v}^{2}}{A^{2}}+\frac{g_{, v}}{g} \frac{A_{, v}}{A}\right) f^{2} \delta^{2}+\ldots(\text { terms at most linear in } \delta) .
\end{aligned}
$$

\section{Appendix E. Coordinate transformations}

In order to express the $\{\hat{u}, \hat{v}, \hat{\theta}, \hat{\phi}\}$ coordinates characterizing the metric (4.6) in terms of $\left\{Y_{1}, Y_{2}, Y_{3}, Y_{4}\right\}$ (which are the coordinates describing (2.21)-(2.30) $)$, we start by inverting (2.15)-(2.17), yielding easily

$$
\begin{gathered}
Y_{0}=\gamma\left(Z_{0}-v Z_{1}\right), \\
Y_{1}=\gamma\left(Z_{1}-v Z_{0}\right), \\
Y_{2}=Z_{2}, \quad Y_{3}=Z_{3}, \quad Y_{4}=Z_{4} .
\end{gathered}
$$

By using (2.3)-(2.7) jointly with (E.1)-(E.3) we obtain that

$$
Y_{0}=\gamma\left(\sqrt{a^{2}-r^{2}} \sinh (t / a)-v r \cos \theta\right)
$$

and

$$
\begin{gathered}
Y_{1}=\gamma\left(r \cos \theta-v \sqrt{a^{2}-r^{2}} \sinh (t / a)\right), \\
Y_{2}=r \sin \theta \cos \phi \\
Y_{3}=r \sin \theta \sin \phi \\
Y_{4}=\sqrt{a^{2}-r^{2}} \cosh (t / a) .
\end{gathered}
$$

Thus, bearing in mind that Eq. (1.14) allows us to get rid of the $Y_{0}$ coordinate, if we want to obtain $\{t, r, \theta, \phi\}$ coordinates of Schwarzschild-de Sitter metric (1.12) 
as functions of $\left\{Y_{1}, Y_{2}, Y_{3}, Y_{4}\right\}$ we have to invert relations (E.5) E.8 . First of all, by exploiting (2.15) -(2.17), the condition $r^{2}=\left(Z_{1}\right)^{2}+\left(Z_{2}\right)^{2}+\left(Z_{3}\right)^{2}$ becomes

$$
r^{2}=\gamma^{2}\left(v \sqrt{\sigma}+Y_{1}\right)^{2}+\left(Y_{2}\right)^{2}+\left(Y_{3}\right)^{2},
$$

whereas on using (E.6) and (E.7) we obtain

$$
r^{2}=\frac{\left(Y_{2}\right)^{2}+\left(Y_{3}\right)^{2}}{\sin ^{2} \theta},
$$

therefore a comparison of (E.9) and (E.10) yields

$$
\sin ^{2} \theta=\frac{\left(Y_{2}\right)^{2}+\left(Y_{3}\right)^{2}}{\gamma^{2}\left(v \sqrt{\sigma}+Y_{1}\right)^{2}+\left(Y_{2}\right)^{2}+\left(Y_{3}\right)^{2}},
$$

whose solutions are given by

$$
\begin{gathered}
\theta=\mp \arcsin \left(\sqrt{\frac{\left(Y_{2}\right)^{2}+\left(Y_{3}\right)^{2}}{\gamma^{2}\left(v \sqrt{\sigma}+Y_{1}\right)^{2}+\left(Y_{2}\right)^{2}+\left(Y_{3}\right)^{2}}}\right)+2 \pi n, \quad(n \text { integer }), \\
\theta=\pi \mp \arcsin \left(\sqrt{\frac{\left(Y_{2}\right)^{2}+\left(Y_{3}\right)^{2}}{\gamma^{2}\left(v \sqrt{\sigma}+Y_{1}\right)^{2}+\left(Y_{2}\right)^{2}+\left(Y_{3}\right)^{2}}}\right)+2 \pi n, \quad(n \text { integer }),
\end{gathered}
$$

therefore at this stage from (E.8) we straightforwardly obtain the relations for $t$, i.e.

$$
t=a\left[\mp \operatorname{arccosh}\left(\frac{Y_{4}}{\sqrt{a^{2}-r^{2}}}\right)+2 \pi i n\right], \quad(n \text { integer }),
$$

and eventually from (E.7) we get

$$
\begin{gathered}
\phi=\arcsin \left(\frac{Y_{3}}{r \sin \theta}\right)+2 \pi n, \quad(n \text { integer }), \\
\phi=\pi-\arcsin \left(\frac{Y_{3}}{r \sin \theta}\right)+2 \pi n, \quad(n \text { integer }) .
\end{gathered}
$$

Thus, Eqs. (E.9), (E.12) and (E.13), (E.14), (E.15) and (E.16), represent the relations which link $\{t, r, \theta, \phi\}$ to $\left\{Y_{1}, Y_{2}, Y_{3}, Y_{4}\right\}$ coordinates. By exploiting these relations, it is possible to express the $\{\hat{u}, \hat{v}, \hat{\theta}, \hat{\phi}\}$ coordinates as functions of $\left\{Y_{1}, Y_{2}, Y_{3}, Y_{4}\right\}$ and to define the equivalence between the boosting procedure and the coordinate shift method.

\section{References}

[1] R. C. Tolman, Relativity, Thermodynamics and Cosmology (Oxford University Press, New York, 1934)

[2] P. C. Aichelburg and R. U. Sexl, On the gravitational field of a massless particle, Gen. Rel. Grav. 2, 303 (1971).

[3] S. Antoci and D. E. Liebscher, Reconsidering Schwarzschild's original solution, Astron. Nachr. 322, 137 (2001). 
[4] I. M. Gel'fand and G. E. Shilov, Generalized Functions, Vol. 2: Spaces of Fundamental and Generalized Functions (Academic Press, New York, 1964).

[5] V. Ferrari and P. Pendenza, Boosting the Kerr metric, Gen. Rel. Grav. 22, 1105 (1990).

[6] C. O. Loustó and N. Sánchez, The ultrarelativistic limit of the boosted Kerr-Newman geometry and the scattering of spin 1/2 particles, Nucl. Phys. B 383, 377 (1992).

[7] M. Hotta and M. Tanaka, Shock wave geometry with nonvanishing cosmological constant, Class. Quantum Grav. 10, 307 (1993).

[8] H. Balasin and H. Nachbagauer, The ultrarelativistic Kerr geometry and its energymomentum tensor, Class. Quantum Grav. 12, 707 (1995).

[9] H. Balasin and H. Nachbagauer, Boosting the Kerr geometry into an arbitrary direction, Class. Quantum Grav. 13, 731 (1996).

[10] J. Podolský and J. B. Griffiths, Impulsive gravitational waves generated by null particles in de Sitter and anti-de Sitter backgrounds, Phys. Rev. D 56, 4756 (1997).

[11] J. Podolský and J. B. Griffiths, Boosted static multipole particles as sources of impulsive gravitational waves, Phys. Rev. D 58, 124024 (1998).

[12] J. Podolský and M. Ortaggio, Symmetries and geodesics in (anti-)de Sitter spacetimes with nonexpanding impulsive waves, Class. Quantum Grav. 18, 2689 (2001).

[13] G. Esposito, R. Pettorino, and P. Scudellaro, On boosted spacetimes with cosmological constant and their ultrarelativistic limit, Int. J. Geom. Methods Mod. Phys. 4, 361 (2007).

[14] I. Y. Aref'eva, Catalysis of black holes/wormholes formation in high energy collisions, Theor. Math. Phys. 161, 1647 (2009).

[15] S. S. Gubser, S. S. Pufu, and A. Yarom, Entropy production in collisions of gravitational shock waves and of heavy ions, Phys. Rev. D 78, 066014 (2008).

[16] A. Taliotis, Black topology production in extra dimensions, Phys. Rev. D 86, 064034 (2012).

[17] M. Anber and L. Sorbo, Two gravitational shock waves on the AdS(3)brane, J. High Energy Phys. 10 (2007) 072.

[18] K. Sfetsos, Fundamental branes and shock waves, Nucl. Phys. B 726, 1 (2005).

[19] N. Kaloper, Gravitational shock waves and their scattering in brane-induced gravity, Phys. Rev. D 71, 086003 (2005).

[20] I. Y. Aref'eva, High-energy scattering in the brane world and black hole production, Part. Nucl. 31, 169 (2000).

[21] I. Y. Aref'eva, A. A. Bagrov, and E. A. Guseva, Critical formation of trapped surfaces in the collision of non-expanding gravitational shock waves in de Sitter spacetime, J. High Energy Phys. 12 (2009) 009.

[22] I. Y. Aref'eva, A. A. Bagrov, and L. V. Joukovskaya, Critical trapped surfaces formation in the collision of ultrarelativistic charges in (A)dS, J. High Energy Phys. 3 (2010) 002.

[23] A. D. Vidal and M. A. Vazquez-Mozo, A note on the collision of Reissner-Nordström gravitational shock waves in AdS, Phys. Lett. B 713, 500 (2012).

[24] S. B. Giddings and D. Marolf, A Global picture of quantum de Sitter space, Phys. Rev. D 76, 064023 (2007).

[25] C. W. Misner, K. S. Thorne, and J. A. Wheeler, Gravitation (W. H. Freeman and Company, San Francisco, 1973).

[26] J. F. Colombeau, Multiplication of Distributions: a Tool in Mathematics, Numerical Engineering and Theoretical Physics (Springer, New York, 1993).

[27] T. Dray and G. 't Hooft, The gravitational shock wave of a massless particle, Nucl. Phys. B 253, 173 (1985).

[28] K. Sfetsos, On gravitational shock waves in curved spacetimes, Nucl. Phys. B 436, 
$721(1995)$

[29] R. Penrose in General relativity: papers in honour of J. L. Synge, ed. L. O' Raifeartaigh (Clarendon, Oxford, 1972).

[30] R. M. Wald, General Relativity (The University of Chicago Press, Chicago, 1984).

[31] S. W. Hawking and G. F. R. Ellis, The Large Scale Structure of Space-Time (Cambridge University Press, Cambridge, 1973).

[32] A. Coley, S. Hervik, and N. Pelavas, Spacetimes characterised by their curvature invariants, Class. Quantum Grav. 26, 025013 (2009).

[33] C. Cherubini, D. Bini, S. Capozziello, and R. Ruffini, Second Order Scalar Invariants of the Riemann Tensor: Applications to Black Hole Spacetimes, Int. J. Mod. Phys. D 11, 06 (2002).

[34] R. P. Geroch, What is a singularity in general relativity?, Ann. Phys. 48, 3 (1968).

[35] B. G. Schmidt, A new definition of singular points in general relativity, Gen. Rel. Grav. 1, 269 (1971).

[36] J. A. Thorpe, Curvature invariants and spacetime singularities, J. Math. Phys. 18, 960 (1977).

[37] R. P. Geroch, Limits of spacetimes, Commun. Math. Phys. 13, 3 (1969).

[38] A. Almheiri, D. Marolf, and J. Sully, Black holes: complementarity or firewalls?, J. High Energy Phys. 02 (2013) 062.

[39] S. L. Braunstein, S. Pirandola, and K. Życzkowski, Better late than never: information retrieval from black holes, Phys. Rev. Lett. 110, 101301 (2013).

[40] S. L. Braunstein and S. Pirandola, Post-firewall paradoxes, arXiv:1411.7195v2 (2015).

[41] L. Susskind, L. Thorlacius, and J. Uglum, The stretched horizon and black hole complementarity, Phys. Rev. D 48, 3743 (1993).

[42] C. R. Stephens, G. 't Hooft, and B. F. Whiting, Black hole evaporation without information loss, Class. Quantum Grav. 11, 621 (1994).

[43] M. Nakahara, Geometry, Topology and Physics (Institute of Physics Publishing, Bristol, 2003).

[44] E. Cartan, On Manifolds with Affine Connection and the Theory of General Relativity (Bibliopolis, Naples, 1986).

[45] B. S. DeWitt in Relativity, Groups and Topology II, eds. B. S. DeWitt and R. Stora (North-Holland, Amsterdam, 1984). 